Article

\title{
Investigation on the Performance Characteristics of 2-Stroke Heavy Fuel Light Aeroengine (2SHFLA) with Different Fuel Injection Systems: Modeling and Comparative Simulation
}

\author{
Yuan Qiao ${ }^{1}$, Li Lin ${ }^{1}$, Wei Zhong ${ }^{1}$ and Kaisheng Huang ${ }^{1,2,3, *(1)}$ \\ 1 State Key Laboratory of Automotive Safety and Energy, School of Vehicle and Mobility, Tsinghua University, \\ Beijing 100084, China; qiao-y17@mails.tsinghua.edu.cn (Y.Q.); linli19@mails.tsinghua.edu.cn (L.L.); \\ weizhong@mail.tsinghua.edu.cn (W.Z.) \\ 2 The Joint Laboratory for Internet of Vehicles, \\ Ministry of Education-China Mobile Communications Corporation, Beijing 100084, China \\ 3 Collaborative Innovation Center of Electric Vehicles in Beijing, Beijing 100081, China \\ * Correspondence: huangks@tsinghua.edu.cn; Tel.: +86-138-0123-7852
}

Received: 8 August 2020; Accepted: 23 September 2020; Published: 2 October 2020

\begin{abstract}
Extensive application of small and medium-sized unmanned aerial vehicles (UAVs) have already made the development of corresponding power system a research hotspot nowadays. Two-stroke heavy fuel light aeroengine (2SHFLA) is selected as the research focus in this paper. The working principle of 2SHFLA with different fuel injection systems is elaborated systematically. By dividing the initial prototype engine into several subsystems, the simulation platform is set up with its key model parameters accurately calibrated against the test data. Simulation platforms of the other two types of engines are subsequently constructed based on the pre-calibrated simulation platform of the initial prototype engine. Afterwards, comparative simulation is performed and the corresponding simulation results include: (1) comparison of performance characteristics of the initial prototype engine fueled with regular gasoline/heavy fuel; (2) comprehensive comparison of the performance characteristics of all the three types of engine.
\end{abstract}

Keywords: performance characteristics; 2SHFLA; injection systems; simulation platform modeling

\section{Introduction}

In recent years, with the great progress of wireless communication technology, flight control technology, and information processing technology, the field of general aviation power has seen great strides in development [1,2]. As a typical representative, small and medium-sized UAVs (maximum take-off weight is generally below $400 \mathrm{~kg}$, maximum mission payload is generally below $60 \mathrm{~kg}$ ) are widely applied due to their advantages of low manufacturing and maintenance costs, excellent portability and usability, and high compatibility, especially in the military flight profiles [2].

With respect to small and medium-sized UAVs, its main functions generally include battlefield surveillance and reconnaissance, real-time relay communication, and continual border patrol. Taking into account these function features, take-off weight and loitering time are often considered as the core indicators for evaluating the performance of small and medium-sized UAVs [3]. Consequently, crucial requirements including high power density, light weight, and compact structure have been met when designing the power systems for small and medium-sized UAVs [4,5]. As a new form of propulsion, 2-stroke heavy fuel light Aeroengine (2SHFLA) is selected as our research focus for its superiority in fulfilling the above-mentioned demands of power systems of small and medium UAVs. 
Technically, 2SHFLA can be recognized as the confluence of a 2-stroke reciprocating piston engine and heavy fuel engine [6]. From the perspective of power type, piston engine has gradually given way to jet engine in the aviation industry since the end of World War II. As a kind of typical jet engine, gas turbine engine is widely applied in various aircraft types because of its particular advantages in thrust-weight ratio [7]. Some small gas turbine engines are also selected as the power plants for UAVs early in their development [8,9]. Generally, the indispensable parts of a gas turbine engine include compressor, combustor, and compressor-driving turbine. Relatively complicated structure makes it much difficult for the realization of lightweight design [10]. Nevertheless, with the growing needs of remotely piloted light aircrafts, small and medium-sized UAVs saw significant and increasing use in the 20th century [8-10]. The miniaturization tendency of UAVs puts forward higher demand for the downsizing of power systems, which strongly limits the application of gas turbine engines and conversely supports the rejuvenation of piston engine [11]. In addition, small turbine engines suffer from an inability to loiter at low enough speed unless a rotary wing UAV is used, but even then the high specific fuel consumption, particularly for small gas turbines, is generally prohibitive [12]. Taking a 2-stroke reciprocating piston engine as an example, it has both higher propulsive efficiency and lower fuel consumption under low and medium flight speed condition (which is also the common flight operation condition of UAVs) when compared with gas turbine engine, thus making it a better choice for small and medium-sized UAVs [8-11].

From the perspective of power fuel, an aerial piston engine has been fueled with regular gasoline for a long time [13]. However, the flash point of regular gasoline is quite low $\left(-45^{\circ} \mathrm{C}-25^{\circ} \mathrm{C}\right)$, which makes it relatively inflammable and explosible. Therefore, regular gasoline is greatly restricted in scenarios demanding high safety coefficients. In contrast, the flash point of heavy fuel (including aviation kerosene and light diesel) is generally $35{ }^{\circ} \mathrm{C}-51^{\circ} \mathrm{C}$, which makes it safer than regular gasoline in the process of fuel storage and transport [14]. Additionally, the manufacturing cost of kerosene is comparatively low considering its widespread application in the current aviation industry [13]. Aviation kerosene and light diesel can also temporarily serve as the fuel supply for land vehicles, showing its good universality $[15,16]$. Based on the above reasons, the transition from regular gasoline to heavy fuel can be observed in the research field of power fuel for small and medium-sized UAVs [17].

However, the physicochemical properties of heavy fuel (high viscosity and low volatility) make it worse in fuel spray quality than regular gasoline under the same engine operation conditions, thus it is more difficult for heavy fuel engine to deliver the fresh charge of homogeneous air-fuel mixture when compared with gasoline engine. The phenomenon leads to inevitable performance degradation of both power and fuel economy for engine supplied with heavy fuel; this is the so-called "power attenuation" problem $[17,18]$. Consequently, necessary modifications need to be made about the fuel injection system in order to address the problem accordingly.

The Orbital Engine Company pioneered the development of air assisted direct injection (AADI) system in 1990s and widened its application field [19-21]. Apart from typical automotive and motorcycle applications, a 2-stroke marine engine is also one of various application scenarios for Orbital AADI systems [19]. As shown in Figure 1, the basic AADI system comprises an outwardly opening air injector, a conventional injection for multi-point port injection (MPI), and an inside premix chamber which connects the air and fuel circuit [21]. The conventional MPI injector provides the fuel metering function and the metered fuel is combined with compressed air in the premix chamber. Finally, the premixed air-fuel charge is directly injected into the cylinder by the air injector before piston reaches the top dead center (TDC). Considering that the compressed airflow continues to expand and accelerate after leaving the injector nozzle, its supersonic aerodynamic force will overcome both the internal friction and surface tension of fuel, which significantly promotes the breakup and atomization process of fuel mixed with the airflow. 


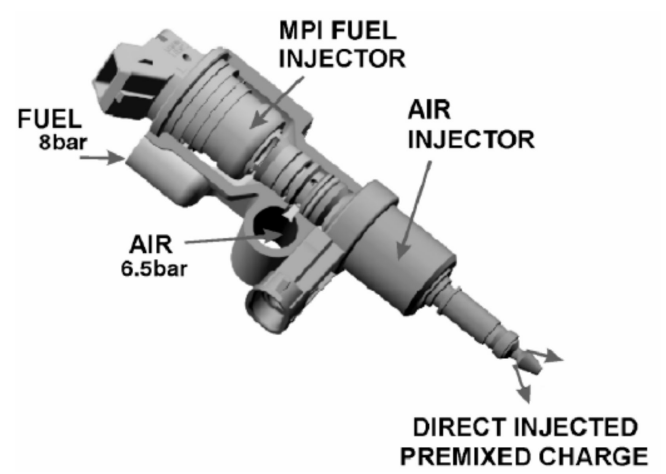

(a)

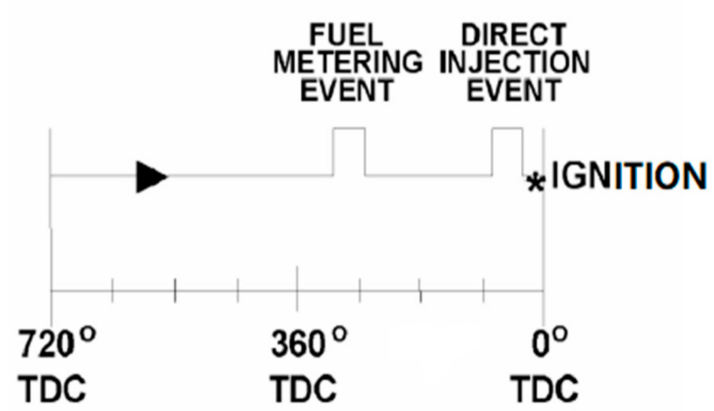

(b)

Figure 1. Schematic of AADI system (a) Injector structure and (b) Injection sequencing.

In the early stages, AADI system was merely applied to gasoline engine for purpose of improving fuel economy [22]. Cathcart et al. demonstrated that the improvement effect of fuel spray quality showed low dependence on the specific type of fuel [23]. The spray droplet size of less-volatile fuel such as aviation kerosene could be also reduced with the atomization uniformity improving, which indicated that the performance attenuation problem caused by supplying engine with less-volatile heavy fuel was alleviated by applying the AADI system. These findings laid the foundation for the application of the AADI system to a heavy fuel engine and stimulated an upsurge of research interest in the related field. Groenewegen et al. discussed the results of continuation of experimentation in utilizing heavy fuels such as JP-8 and D2 diesel to satisfy the necessary power requirements of a $33.5 \mathrm{cc}$ engine equipped with an AADI system [24]. Indicated specific fuel consumption (ISFC) and brake specific fuel consumption (BSFC) were observed as a measure of performance and emissions data was gathered for different kinds of fuels. Preliminary results indicated that heavy fuels and heavy biofuels could meet power requirements while lowering fuel consumption. Koci et al. extended air-assist feasibility understanding to high-pressure environments by using a combination of analytical zero-dimensional (0-D) theory and three-dimensional (3D) computational fluid dynamics (CFD) [25]. Analyses were completed and carried out for traditional high-pressure fuel-only, internal air-assist, and external air-assist fuel-air mixing processes. La et al. focused on the process of benchmarking a 2-stroke heavy fuel spark ignited engine with an integrated AADI system, outlined the setup and commissioning phases of testing, and discussed design features and performance attributes of both the investigated engine and AADI system [26]. Gao et al. performed the systemic experimental study of a 2-stroke engine fueled with aviation kerosene through using visualization tools including a high-speed camera and a phase Doppler particle analyzer. The effects of ambient pressure, temperature, and control parameters on spray width, penetration, spatial diffusion area, and Sauter Mean Diameter (SMD) were analyzed [27]. In order to research the transient spray characteristics of AADI system, Yang et al. carried out corresponding CFD simulation targeted at transient injection with a different back pressure and injection pressure and established a constant volume bomb test cell to verify the simulation results $[28,29]$. Hu et al. developed a single-cylinder prototype engine with AADI system and investigated the influences of different control parameters including injection timing, injection duration, and injection pressure on the engine combustion characteristics [30].

In general, injection systems play an important role in the heavy fuel engine, and AADI system offers a comparatively mature technical solution [27-30]. However, the AADI system must be used with compressed air source and pressure regulator as auxiliary devices, which sets barriers to lightweight design of 2SHFLA. In addition, the reliability of these sophisticated devices is relatively low when UAVs driven by 2SHFLA with AADI system are flying at high altitude. Taking into account factors including both being overweight and susceptibility to operating environment, the AADI system ceases to be practicable in terms of on-board application for UAVs. Actually, only a ground test has been 
conducted in pertinent literature and few studies have provided trial flight results of UAVs powered by 2SHFLA with AADI system.

In this paper, a 2-stroke light aeroengine originally designed to run on regular gasoline is thoroughly studied. Based on this initial prototype engine, the simulation platform and experiment platform are established subsequently. Considering the 2SHFLA scenario, another two types of engine schemes are put forward in order to improve the performance characteristics of the engine when supplied with heavy fuel. Based on the accurately-calibrated simulation platform of the initial prototype engine, simulation platforms of the other two engines are also constructed. Comparative simulation is carried out with a comprehensive analysis of simulation results performed.

\section{Fundamentals of the Investigated Engine}

\subsection{Working Principle of the Initial Prototype Engine}

A horizontally opposed 2-cylinder 2-stroke boxer engine originally designed to run on regular gasoline is selected as the initial prototype engine. Figure 2 displays its overall look. The main specifications of the initial prototype engine are presented in Table 1. As mentioned in Section 1, a 2-stroke engine type brings distinctive benefits of compact structure and high power density. In addition, the boxer engine shows a unique advantage in self-balance due to its highly symmetrical structure. These reasons make the initial prototype engine suitable as the power unit of small and medium-sized UAVs.

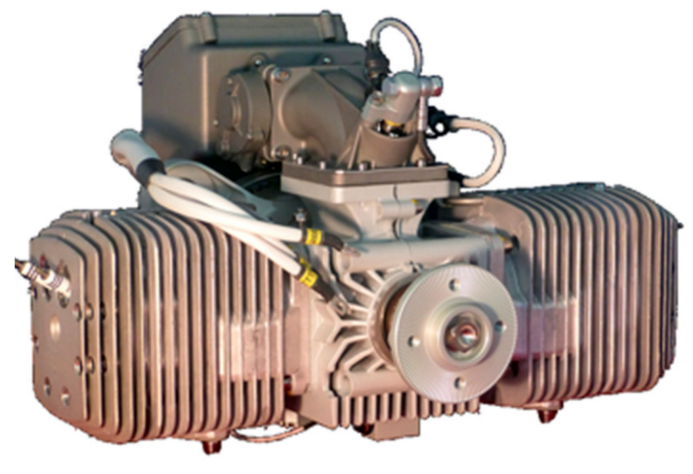

(a)

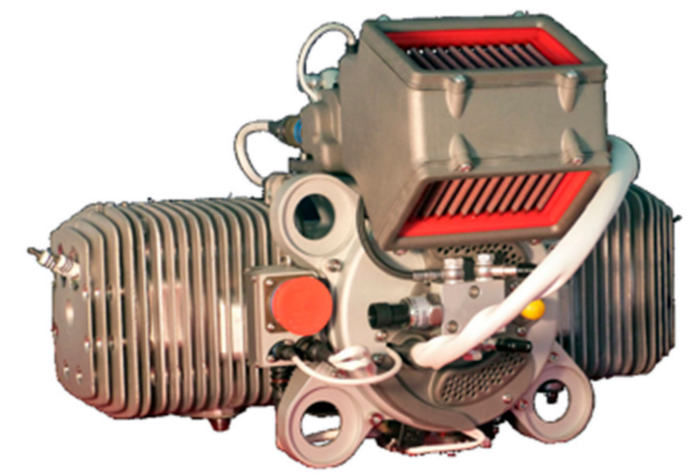

(b)

Figure 2. Overall look of the initial prototype engine (a) Front view and (b) Back view.

As listed in Table 1, the injection system applied in the initial prototype engine is conventional port fuel injection (PFI). Figure 3 is a schematic diagram of its basic working principle. Actually, some sectional drawings of the initial prototype engine can be obtained based on the 3D engine model. However, the geometric structure of the actual research engine is fairly sophisticated, and it needs many large images to display the structure, which takes up too much space in this paper. Considering that, specific sectional drawings are not presented here and a basic diagram is enough to illuminate the working principle. When fresh air flows into the intake pipe, it will first pass through the throttle valve. An electronically-controlled injector located behind the throttle valve will inject fuel into the intake pipe at an appropriate time. The injected fuel collides and blends with the intake airflow, continually accelerates its atomization and evaporation process, and gradually promotes the formation of homogeneous air-fuel mixture. The intake pipe after the throttle valve is separated from the crankcase by a reed valve. When the crankcase pressure drops to a certain value, the reed valve opens to guide the fresh charge of air-fuel mixture into the crankcase. As the fresh charge enters the crankcase, the crankcase pressure gradually goes up. When the pressure difference between the intake pipe and the crankcase drops below the critical threshold of reed valve, it will return to the closed state, 
separating the intake pipe from the crankcase again. The fresh charge of air-fuel mixture is completely contained in the closed volume composed of the crankcase and the scavenging passage, waiting to be sent into the cylinder after the scavenging process starts.

Table 1. Main specifications of the initial prototype engine.

\begin{tabular}{cc}
\hline Parameters & Value \\
\hline Engine Type & horizontally opposed 2-cylinder 2-stroke \\
Injection System & port fuel injection (PFI) \\
Ignition System & double programmed inductive discharging system \\
Intake system & naturally aspirated \\
loop scavenging \\
Scavenging System & forced air cooling \\
Cooling System & mixed lubrication $(2 \%$ synthetic oil) \\
Lubrication System & $0.500 \mathrm{~L}$ \\
Total Displacement & $18.6 \mathrm{~kg}$ \\
Total Weight & $75 \mathrm{~mm}$ \\
Bore & $56 \mathrm{~mm}$ \\
Stroke & 10.6 \\
Compression Ratio & $6500 \mathrm{r} / \mathrm{min}$ \\
Rated Speed & $42 \mathrm{~N} \cdot \mathrm{m}$ \\
Rated Torque & $28.6 \mathrm{~kW}$ \\
Rated Power & $6800 \mathrm{r} / \mathrm{min}$ \\
Maximum Safety Speed & $2200 \mathrm{r} / \mathrm{min} \sim 2800 \mathrm{r} / \mathrm{min}$ \\
Minimum Steady Speed & $-20^{\circ} \mathrm{C}-65^{\circ} \mathrm{C}$ \\
Operating Temperature & $1000 \mathrm{~h}$ \\
Time Between Overhaul &
\end{tabular}

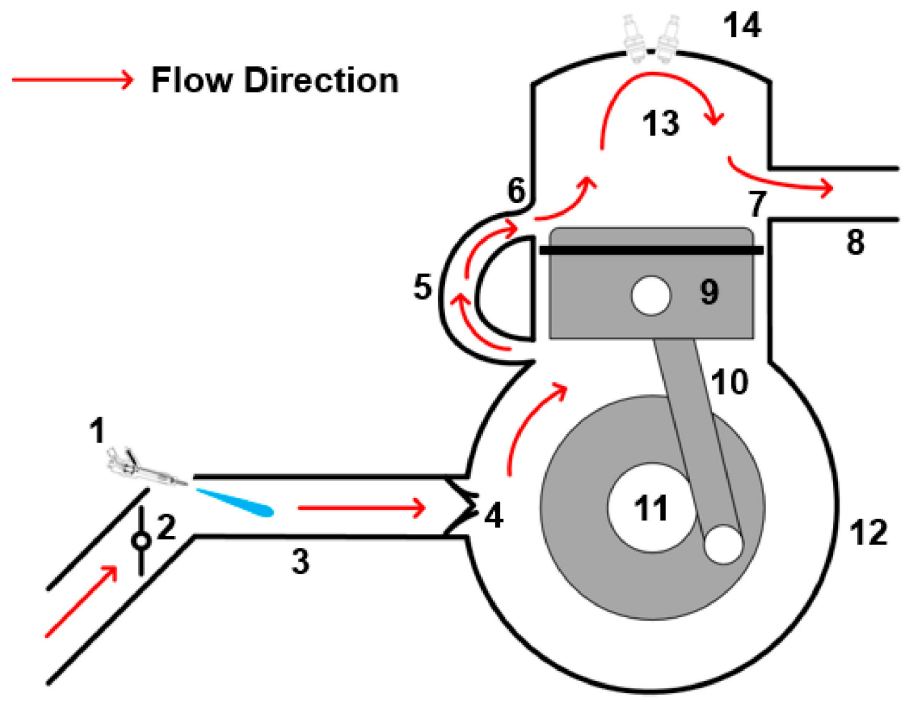

1- Injector

2- Throttle Valve

3- Intake Pipe

4- Reed Valve

5- Scavenging Passage

6- Scavenging Port

7- Exhaust Port

8- Exhaust Pipe

9- Piston

10- Connecting Rod

11- Crankshaft

12- Crankcase

13- Cylinder

14- Spark Plugs

Figure 3. Schematic diagram of the initial prototype engine.

As one of the most distinguishing characteristics of a 2-stroke engine, the intake and exhaust valve timing is determined by the specific positions of scavenging port/exhaust port on the cylinder wall. Detailed configurations of the intake and exhaust valve timing of the initial prototype engine is shown in Table 2. With regard to the initial prototype engine, the unique gas exchange process features prominently in its working principle. As shown in Figure 4, the complete gas exchange process can be roughly divided into three phases including free exhaust phase, forced scavenging phase, and final exhaust phase [31,32]. The expansion stroke of the initial prototype engine gradually draws to a close as the piston descends from the TDC. When the piston uncovers the exhaust port, a free exhaust phase starts and the cylinder begins to blow down its pressure to the exhaust pipe. During this phase, burned gases flow out through the exhaust port and the cylinder pressure sees a 
drastic decrease. Burned gases discharged in this phase accounts for approximately $70-80 \%$ of the total burned gas discharged during the complete gas exchange process. Subsequently, the piston continues go down, compressing the air-fuel mixture contained in the crankcase volume further. The forced scavenging phase is started by the piston uncovering the scavenging port. It should be noted that the combination of piston descending and burned gases leaving leads to the increase of vacuum in the cylinder. With the scavenging port opening, air-fuel mixture filled in the crankcase volume will be pumped into the cylinder through the scavenging passage. During the forced scavenging phase, residual burned gases is driven out of cylinder by the influx of air-fuel mixture and there exists an overlap of intake phase and exhaust phase. The piston starts to move up after it reaches the bottom dead center (BDC). As shown in Table 2, the intake and exhaust valve timing are symmetrical relative to the BDC. Considering that the piston uncovers the exhaust port $\left(95^{\circ} \mathrm{CA}\right)$ earlier than scavenging port $\left(114^{\circ} \mathrm{CA}\right)$, the piston will seal off the scavenging port $\left(246^{\circ} \mathrm{CA}\right)$ earlier than scavenging port in the compression stroke. The final exhaust phase starts after the closing of scavenging port. The in-cylinder gases are composed of the fresh charge of air-fuel mixture and residual burned gases that have not been discharged yet. During this phase, the exhaust port is still open. This process will last for a while until the exhaust port is completely closed, which puts an end to the final exhaust phase.

Table 2. Intake and exhaust valve timing of the initial prototype engine.

\begin{tabular}{cc}
\hline Parameters & Value \\
\hline Exhaust Port Open & $95^{\circ} \mathrm{CA}$ \\
Scavenging Port Open & $114^{\circ} \mathrm{CA}$ \\
Scavenging Port Close & $246^{\circ} \mathrm{CA}$ \\
Exhaust Port Close & $265^{\circ} \mathrm{CA}$ \\
\hline
\end{tabular}

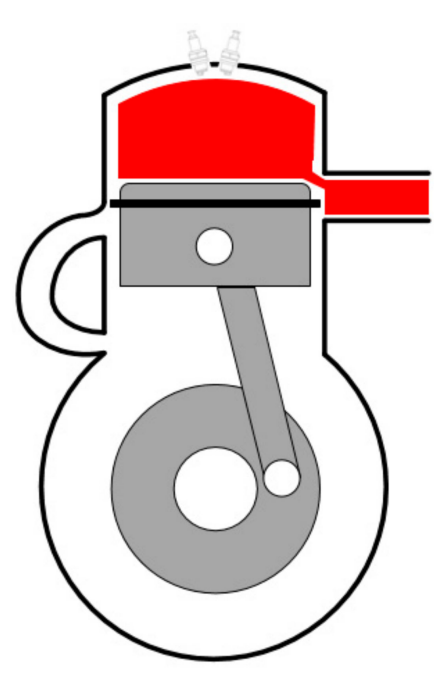

Free Exhaust Phase

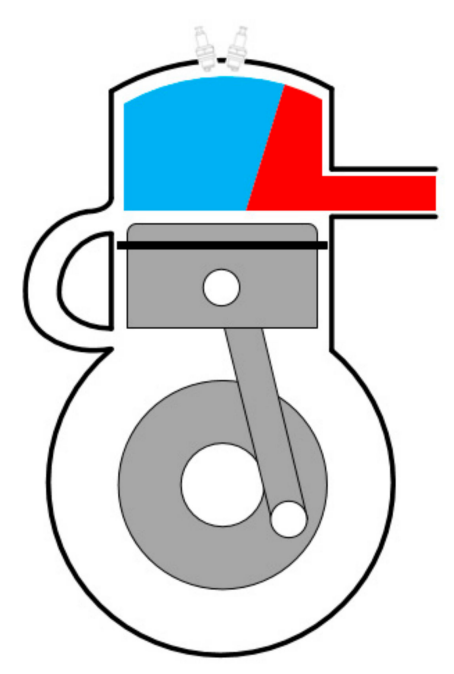

Forced Scavenging Phase

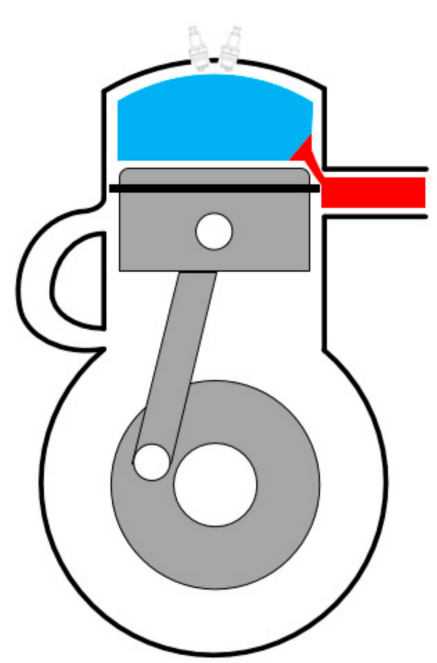

Final Exhaust Phase

Figure 4. Schematic diagram of the gas exchange process of the initial prototype engine.

\subsection{Working Principle of the Engine in the Relevant Reference (Reference Engine)}

The physicochemical properties of heavy fuel significantly distinguish from regular gasoline. High viscosity and low volatility make the atomization and evaporation process of heavy fuel more difficult under the same injection condition, resulting in worse homogeneity of air-fuel mixture and the decline in engine power. As a consequence, necessary modifications need to be made about the fuel injection system to address this "power attenuation" problem. As mentioned in Section 1, a traditional AADI system utilizes the supersonic aerodynamic force of compressed air leaving the nozzle to overcome the internal friction and surface tension of heavy fuel. During the injection process, fuel droplets violently impact against the intake airflow and gradually breaks up into smaller ones, 
paving the way for the formation of homogeneous air-fuel mixture. However, the AADI system must be supported by indispensable but sophisticated auxiliary devices including compressed air source and pressure regulator. Considering that UAVs powered by 2SHFLA often cruise at altitudes up to $4000 \mathrm{~m}$, there is no guarantee for the reliability of integrated AADI system, which greatly limits its on-board application in UAVs.

William of Design \& Manufacturing Solutions Inc. transplanted the designing concept of resonant intake/exhaust system (which are widely applied in conventional 4-stroke engine) into 2-stroke engine, originally proposed the scheme of compression wave injection (CWI) system, and provided a feasible solution for the above-mentioned problem [33]. The engine equipped with CWI system is called "reference engine" to be differentiated from the initial prototype engine.

Figure 5 displays the working principle of the reference engine. Compared with the initial prototype engine, a conventional PFI system is replaced by a CWI system in the reference engine, so that fuel is no longer injected into the intake pipe. Instead, a fuel metering system is connected to a close-end tube (CWI tube) mounted outside the cylinder, and fuel is directly injected into the CWI tube. A connecting orifice is machined through the cylinder wall, and it leads to the CWI tube. During the expansion stroke, the piston moves toward the exhaust opening position and most of the burned gases in the cylinder are discharged through the exhaust port. The position of the connecting orifice is just below the exhaust port. As the piston continues down its way, the connecting orifice is subsequently uncovered. It should be noted that the orifice is positioned such that the cylinder pressure is still much higher than the CWI tube pressure at the orifice open timing. Huge pressure difference causes a strong right moving compression wave to enter the CWI tube, along with an associated influx of burned gases from the cylinder into the CWI tube. For one thing, fuel has been metered into the CWI tube near the connecting orifice before the orifice open timing. Considering that these in-flowing high-temperature gases are introduced to the same position as the fuel, they are able to pre-vaporize the latter and thus improve the quality of heavy fuel atomization. In addition, the above-mentioned right moving compression wave travels down the CWI tube and reflects off its closed end, the reflected wave is a left moving compression wave. As the left moving wave reaches the connecting orifice, it causes the orifice pressure to significantly exceed the cylinder pressure and thus generates mass flow from the CWI tube into the cylinder. Consequently, the residual burned gases in the CWI tube return to the cylinder with the injected fuel.

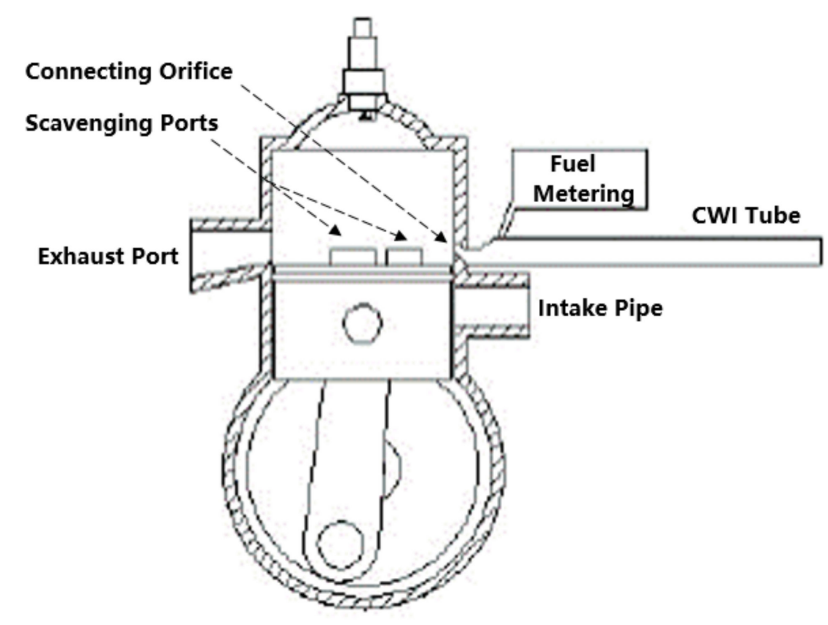

Figure 5. Schematic diagram of the reference engine [33].

Figure 6 displays the correlation between pressure curve and mass flow curve of the connecting orifice in a single cycle. It can be observed that positive mass flow (from the cylinder to the CWI tube) is immediately generated after the orifice open timing. As the piston continues to descend, the cylinder pressure rapidly drops to nearly the same level as the orifice pressure. Accordingly, the mass flow rate 
gradually returns to zero with the slightest perceptible nuance between the orifice pressure and the cylinder pressure. However, the orifice pressure will drastically exceed the cylinder pressure when the reflected compression wave travels back to the connecting orifice, which leads to negative mass flow (from the CWI tube to the cylinder). Consequently, a portion of burned gases previously discharged into the CWI tube goes back to the cylinder, mixed with the injected fuel. After that, the orifice pressure drops once again, and finally it can be seen that positive mass flow is regenerated at about $210{ }^{\circ} \mathrm{CA}$ because of the orifice pressure falling below the cylinder pressure. The mass flow rate returns to zero when the piston reaches the orifice close timing. The cylinder pressure continues to go up with the progress of compression stroke, and the orifice pressure remains at a constant level with the CWI tube sealed.

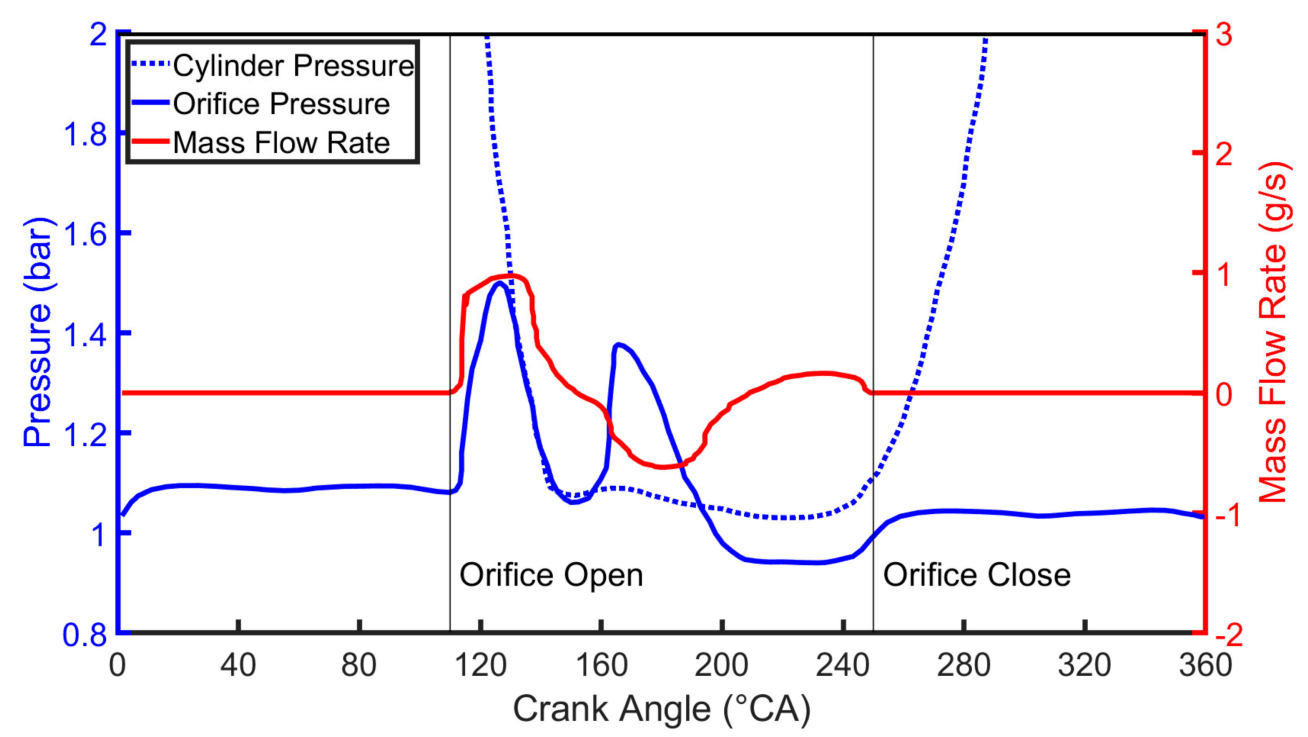

Figure 6. Pressure and mass flow curves of the connecting orifice in a single cycle [33].

In general, the PFI system in the initial prototype engine injects fuel into the intake pipe where both air velocity and air temperature are comparatively low. When the fuel supply is switched from regular gasoline to heavy fuel, it is more difficult to form homogeneous air-fuel mixture and power decrease is inevitable. With regard to the reference engine, the CWI system adjusts the specific position of fuel injection. By bringing in the CWI tube, heavy fuel is injected into the hot environment filled with fast-flowing high-pressure burned gases, which creates favorable conditions for its atomization process and consequently alleviates the "power attenuation" problem of 2SHFLA.

As mentioned above, the connecting orifice needs to be structurally machined on the cylinder wall to connect the cylinder and the CWI tube. For one thing, the specific position of the connecting orifice needs to be accurately restricted between the exhaust port and scavenging ports, which gives rise to processing difficulty. Additionally, the cylinder block of the initial prototype engine adopts a cast aluminum alloy with fine machining, which means that orifice machining will unavoidably cause damage to the surface of cylinder wall more or less. Although some manufacturing facilities are able to drill a hole in a precise position without serious damage, it also brings about a relatively high processing cost. Considering both of the two, it is quite difficult for the initial prototype engine to be retrofitted to one with CWI system without excessive cost or structural impairment.

In addition, William also admits that there is strong potential for the injected fuel to pool in the CWI tube during the period of warm-up, which is very dependent on the engine position [33]. Figure 7 displays the mass flow curve extracted from Figure 6. It can be observed that the curve can be divided into three regions according to the zero mass flow rate line. $S_{1}$ and $S_{3}$ represent positive mass flow (from the cylinder to the CWI tube) and $S_{2}$ represents negative mass flow (from the CWI tube to the cylinder). Mass flow curve in each region can be integrated and corresponding integral values are listed 
in Table 3. Based on Table 3, conclusions can be drawn that there is obvious net inflow $\left(S_{1}+S_{3}>S_{2}\right)$ entering the CWI tube during the opening of the connecting orifice, which indicates that part of the burned gases mixed with injected fuel will be trapped in the CWI tube after the connecting orifice is closed. In other words, only part of the burned gases mixed with injected fuel are sent into the cylinder. Not only is the engine power reduced and fuel consumption increased, but also fuel remaining in the CWI tube is prone to deposit on the tube wall near the connecting orifice. This phenomenon can be more severe during the period of warm-up, which remarkably degrades the cold start performance of 2SHFLA.

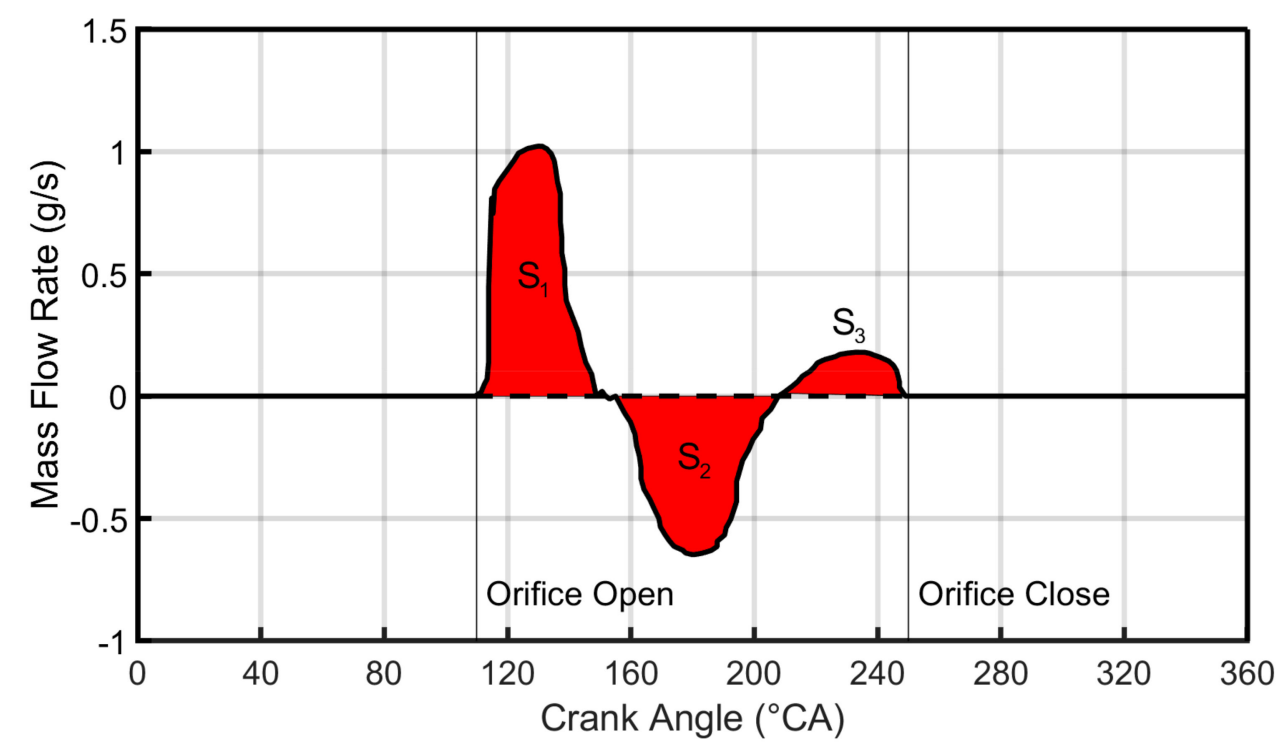

Figure 7. Mass flow curve of the connecting orifice in a single cycle.

Table 3. Integral calculation results of the mass flow curve in different regions.

\begin{tabular}{cc}
\hline Region & Value \\
\hline $\mathrm{S}_{1}$ & $1.196 \mathrm{mg}$ \\
$\mathrm{S}_{2}$ & $0.975 \mathrm{mg}$ \\
$\mathrm{S}_{3}$ & $0.220 \mathrm{mg}$ \\
\hline
\end{tabular}

\subsection{Working Principle of the Retrofitted Engine}

In order to address the fuel pooling problem and reduce the mechanical processing difficulty, a novel engine scheme with scavenging port semi-direct injection (SPSDI) system is proposed. To be differentiated from the above two types of engine, it is called a "retrofitted engine." Based on the reference engine scheme, the retrofitted engine makes full use of the CWI system's unique advantages and shakes off the problem of fuel pooling in the CWI tube simultaneously. Figure 8 is a schematic diagram of the retrofitted engine.

To send the burned gases mixed with injected fuel into cylinder, the reference engine utilizes the dynamic effect caused by compression wave propagation in the CWI tube. This designing concept originated from the resonant intake/exhaust system of 4-stroke engine is also applied to the retrofitted engine. A close-end tube named resonance tube is mounted at the end of the scavenging passage. With the resonance tube playing a role as an inertia supercharger, the retrofitted engine is able to deliver more fresh air into the cylinder and thus improve volumetric efficiency. What makes the retrofitted engine different is that heavy fuel is no longer injected into the tube. Instead, it is directly injected into the cylinder. With regard to the mounting position of injector, it is placed at the junction of scavenging passage and resonance tube. In addition, the axis direction of injector should be in conformity with the inclined direction of scavenging port as to avoid that the fuel spray collides with the internal wall 
of scavenging passage. For one thing, most of the injected fuel will be directed into the upper part of cylinder, where the retained high-temperature burned gases can greatly promote the atomization and evaporation process of the injected fuel. Moreover, scavenging flow from the crankcase can be utilized to flush the scavenging passage and carry the residual fuel retained in the scavenging passage to the cylinder. Compared with the reference engine, the retrofitted engine also greatly improves the atomization quality of heavy fuel while eliminating the fuel pooling problem. Moreover, there is no need to drill a connecting orifice on the cylinder wall, which significantly reduces the processing difficulty and mechanical damage brought to the engine.

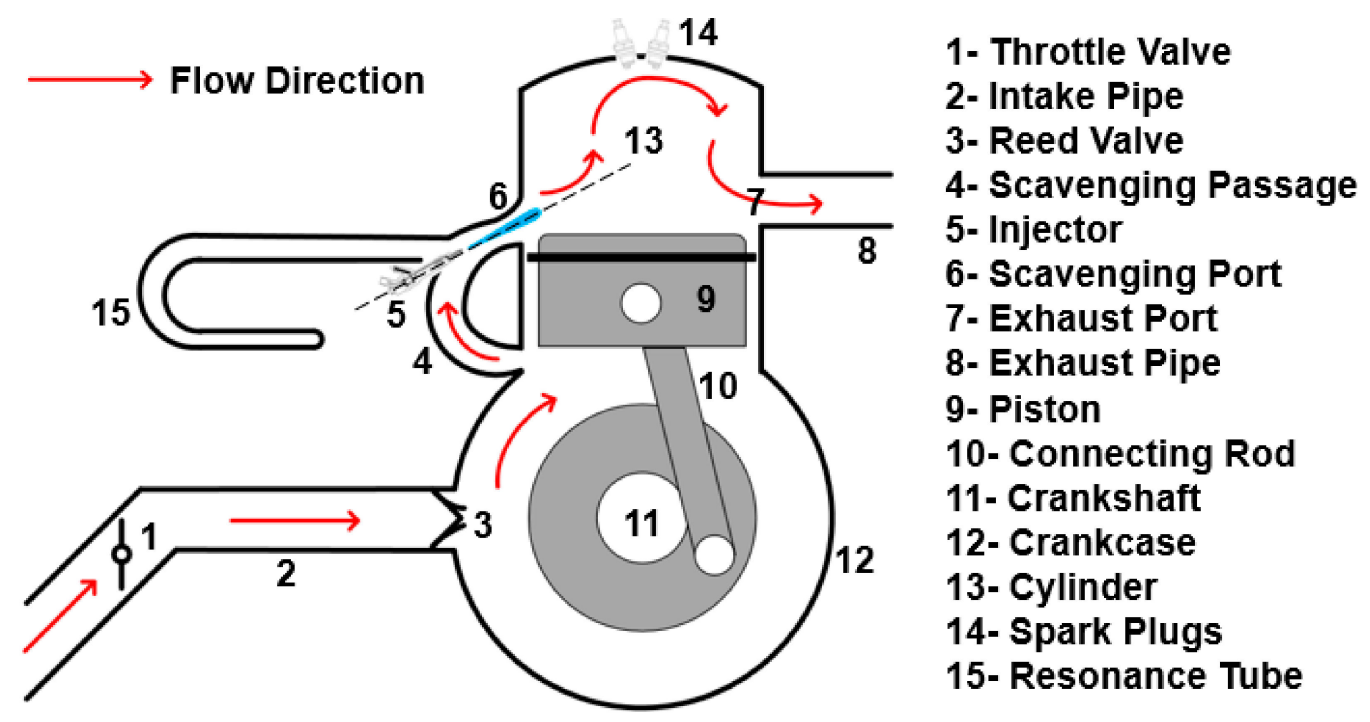

Figure 8. Schematic diagram of the retrofitted engine.

With regard to the retrofitted engine, fuel is directly injected into the cylinder, which is similar to the gasoline direct injection (GDI) engine to some degree. With regard to the Orbital AADI system, the fuel injection pressure is generally low (8 bar) [20]. However, the cylinder injector of GDI engine is mounted at the cylinder head and the injection pressure is generally up to 50-150 bar [34]. As a contrast, the injector of the retrofitted engine is mounted toward the scavenging port and the injection pressure is only $3-5$ bar. In order to distinguish the injection system in this retrofitted engine from conventional GDI system, the former is called "scavenging port semi-direct injection" system. The characteristics of the above-mentioned three types of engine are compared in Table 4. Preliminary comparison shows that the superiority in overall performance retrofitted engine emerges.

Table 4. Characteristics of all the three types of engine.

\begin{tabular}{|c|c|c|c|}
\hline Engine Type & \multirow{2}{*}{ Initial Prototype Engine } & \multirow{2}{*}{ Reference Engine } & \multirow{2}{*}{ Retrofitted Engine } \\
\hline Characteristics & & & \\
\hline Fuel Injection System Type & PFI & CWI & SPSDI \\
\hline Forming Position of Air-Fuel Mixture & induct pipe & CWI tube & cylinder \\
\hline Atomization Quality of Heavy Fuel & poor & good & good \\
\hline Mechanical Processing Difficulty & No & high & low \\
\hline Fuel Pooling Problem & No & Yes & No \\
\hline
\end{tabular}

\section{Simulation and Experiment Platform Construction of the Initial Prototype Engine}

\subsection{Simulation Platform Modeling of the Initial Prototype Engine}

Based on the main specifications of the initial prototype engine, its simulation platform should be established for subsequent research targeted at performance characteristics. Developed by Gamma 
Technologies, GT-SUITE is highly acclaimed for its expertise in field of internal combustion engine simulation $[35,36]$. As a leading multi-physics simulation software package, GT-SUITE supplies a comprehensive set of component libraries from which accurate models of almost any kind of engine can be built. The simulation platform of the initial prototype engine is constructed in the integrated simulation environment GT-ISE provided by GT-SUITE. Considering the structure and functions of the initial prototype engine, it can be divided into five subsystems including intake system, fuel injection system, crank-connecting rod system, cylinders, and exhaust system. The above subsystems are modeled separately and linked to each other appropriately in GT-ISE for purpose of constructing the integrated simulation platform.

\subsubsection{Intake System Modeling}

The intake system of the initial prototype engine mainly consists of atmospheric environment, intake pipe, throttle valve, reed valve, and other parts. The atmospheric environment represents the ambient environment where the initial prototype engine operates, and its temperature, pressure, and composition all have a considerable effect on the intake and combustion process of the engine. The EndEnvironment simulation module is selected to model atmospheric environment with the related parameter settings shown in Table 5. It should be mentioned that these values refer to the standard atmosphere at sea level, but not at altitude.

Table 5. Parameter settings of the simulation module of atmospheric environment.

\begin{tabular}{cc}
\hline Parameter & Value \\
\hline Temperature & $300 \mathrm{~K}$ \\
Absolute Pressure & $1.013 \mathrm{bar}$ \\
Composition & standard air \\
\hline
\end{tabular}

Before entering the intake pipe, the air flow first passes through an air filter to clean itself. GT-ISE provides the dedicated simulation module DustFilter to model this indispensable component in the intake system. Intake pipe is the main flow passage for fresh air in the intake system. The intake pipe of the initial prototype engine is divided into two parts: pipe before the throttle valve and pipe after the throttle valve. To distinguish them, "pipe-1" and "pipe-2" are marked, respectively. Actually, neither of these two pipes are standard round tubes, but appropriate simplifications can be made for this point, and both of the two pipes are regarded as standard round tubes in the modeling process. Therefore, the PipeRound simulation module is selected to model them in GT-ISE.

Throttle valve is of vital importance in the intake system because of its capability of controlling the mass flow of intake air. The variations of throttle valve opening have a direct impact on the flow coefficient, and thus affect its equivalent flow area. Figure 9 displays the variation curve of flow coefficient with throttle valve opening. Predicated on the flow coefficient, the empirical formula for calculating the mass flow rate of throttle valve is listed in the following equations [37]:

$$
\begin{gathered}
\dot{m}_{a t}\left(\alpha, n, p_{\text {pipe-2 }}\right)=\min \left\{\dot{m}_{1}, \dot{m}_{2}\right\} \\
\dot{m}_{1}=C_{11}+C_{12} n \\
\dot{m}_{2}=c_{t} \frac{\pi}{4} D_{\text {th }}^{2} \frac{p_{\text {pipe- }-1} \sqrt{\frac{2 \kappa}{\kappa-1}}}{\sqrt{R T_{\text {pipe- }}}} \beta(\alpha) \beta\left(p_{\text {pipe- } 2}\right)+C_{21} \\
\beta(\alpha)=1-\cos \left[\frac{\pi}{2}\left(\alpha-\alpha_{0}\right)\right]
\end{gathered}
$$




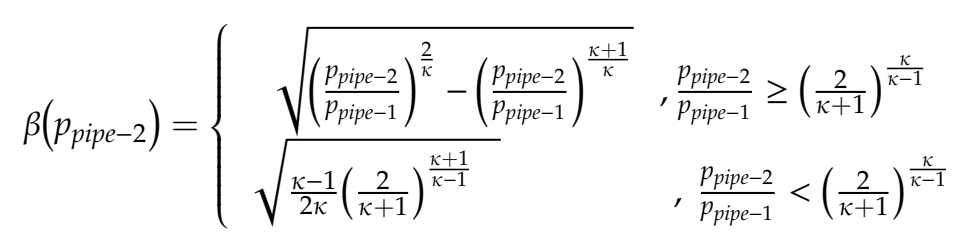

where $\dot{m}_{a t}\left(\alpha, n, p_{\text {pipe-2 }}\right)$ is the mass flow rate of throttle valve and the function of throttle valve opening $\alpha$, engine speed $n$, and the pressure in pipe- $2 p_{\text {pipe-2 }}, c_{t}$ is flow coefficient, $D_{t h}$ is the diameter of throttle valve, $p_{\text {pipe- } 1}$ is the pressure in pipe- $1, T_{\text {pipe- } 1}$ is the temperature in pipe- $1, \kappa$ is the isentropic exponent of working substance, $\dot{m}_{1}, \dot{m}_{2}, \beta(\alpha)$, and $\beta\left(p_{\text {pipe- } 2}\right)$ are all necessary intermediate variables for calculation, $C_{11}, C_{12}, C_{21}$, and $\alpha_{0}$ are all constant coefficients in the corresponding equations. The specialized simulation module ThrottleConn is chosen to model the throttle valve with the variation curve displayed in Figure 9 acting as an indispensable attribute of the simulation module input.

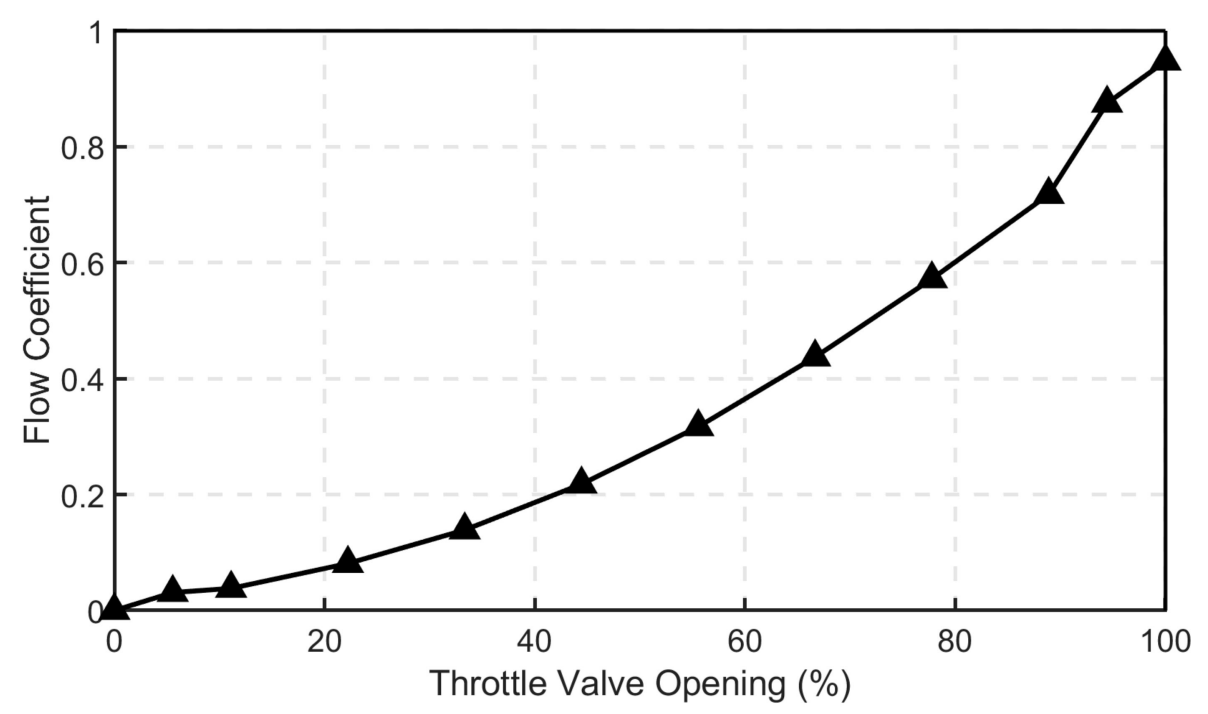

Figure 9. The variation curve of flow coefficient with throttle valve opening.

Reed valve is a check valve located at the end of pipe-2. As mentioned in Section 2.1, the reed valve will open when the pressure difference between the upstream of reed valve (i.e., pipe-2) and the downstream of reed valve (i.e., crankcase) reaches a certain value. The reed valve will close again when the corresponding pressure difference drops to a certain value, and seal off the fresh charge of air-fuel mixture into the crankcase to prevent it from flowing back to the induct pipe. The specialized simulation module ValveCheckConn is selected to model the reed valve with the specific parameter settings shown in Table 6. In Table 6, the parameter settings of reed valve fall into two categories, geometric structure parameters and mechanical property parameters. The former one (valve diameter, upstream pressure area, downstream pressure area, initial valve lift, and maximum valve lift) are determined by accurate measurement, while the latter one (diaphragm mass, spring stiffness, pretension setting length, seat stiffness, and stop stiffness) are obtained according to the product specification provided by the original equipment manufacturer (OEM) of reed valve. Similar to throttle valve, the variation of valve lift of reed valve also directly affects its flow coefficient. Consequently, the variation curve of flow coefficient with valve lift shown in Figure 10 needs to be included within the simulation module input. 
Table 6. Parameter settings of reed valve.

\begin{tabular}{cccc}
\hline Parameter & Value & Parameter & Value \\
\hline Valve Diameter & $45 \mathrm{~mm}$ & Diaphragm Mass & $0.54 \mathrm{~g}$ \\
Upstream Pressure Area & $1569.29 \mathrm{~mm}^{2}$ & Spring Stiffness & $1500 \mathrm{~N} / \mathrm{m}$ \\
Downstream Pressure Area & $1437.38 \mathrm{~mm}^{2}$ & Pretension Setting Length & $10.7 \mathrm{~mm}$ \\
Initial Valve Lift & $0 \mathrm{~mm}$ & Seat Stiffness & $1.0 \times 10^{8} \mathrm{~N} / \mathrm{m}$ \\
Maximum Valve Lift & $8 \mathrm{~mm}$ & Stop Stiffness & $1.0 \times 10^{8} \mathrm{~N} / \mathrm{m}$ \\
\hline
\end{tabular}

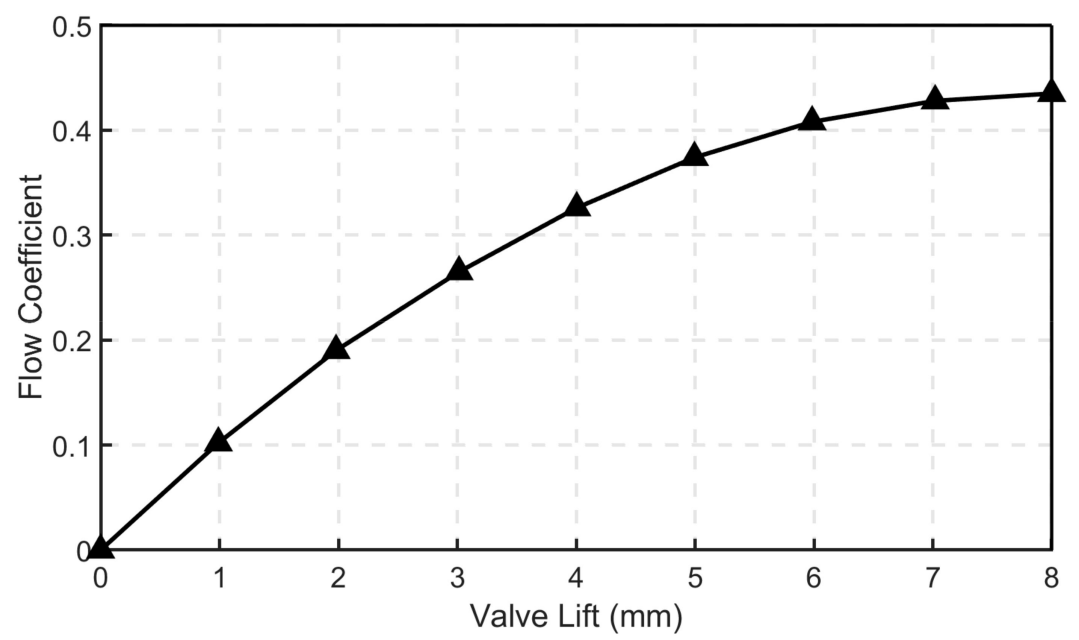

Figure 10. The variation curve of flow coefficient with valve lift.

Actually, there is no so-called "intake manifold" structure in the initial prototype engine. As mentioned in Section 2.1, the initial prototype engine displays a highly symmetrical structure because of its horizontally opposed cylinders. Consequently, the complete crankcase is replaced by two "half-crankcases" in the subsequent modeling process of crank-connecting rod system [38]. The reed valve is connected to two "half-crankcases" and each "half-crankcase" supplies a cylinder with scavenging flow. Therefore, a simulation module responsible for evenly splitting the flowing gases through the reed valve needs to be added at the end of intake system. To some extent, it plays a role similar to intake manifold in the conventional 4-stroke engine. The FlowSplitGeneral simulation module is selected to complete the intake system modeling. Figure 11 displays the layout of intake system in GT-ISE.

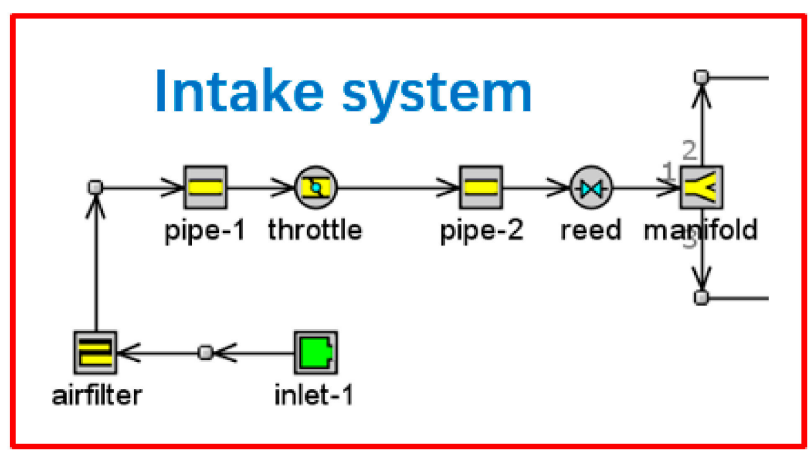

Figure 11. The layout of intake system in GT-ISE.

\subsubsection{Fuel Injection System Modeling}

Considering that the PFI system is adopted by the initial prototype engine, the injector is directly installed on pipe-2. The InjAF-RatioConn module suitable for PFI simulation is chosen to model the 
fuel injection system, and corresponding input parameters are shown in Table 7. It should be noted that the value of injector location is the normalized location along a pipe where injection occurs. 0.0 represents the inlet end of the pipe, and 1.0 the outlet. Considering that the PFI injector is actually installed at the outlet end of pipe-2, the injector location is set as 1.0. With respect to fuel ratio specification, air-fuel ratio is a common option, and it indicates that the quantity of injected fuel is defined in terms of mass of fuel to mass of air. The initial prototype engine is originally designed to run on regular gasoline, thus the injected fluid is set as regular gasoline with a temperature of $300 \mathrm{~K}$ which is equal to ambient temperature. As for the vaporized fluid fraction, it reflects the mass fraction of the injected liquid that will vaporize immediately after injection. Given that the initial prototype engine is a typical PFI engine fueled with regular gasoline, a normal value $30 \%$ is recommended by GT-ISE, and thus adopted as the specific value of this parameter [35].

Table 7. Parameter settings of the simulation module of fuel injection system.

\begin{tabular}{cc}
\hline Parameter & Value \\
\hline Injector Location & 1.0 \\
Fuel Ratio Specification & air-fuel ratio \\
Injected Fluid Type & regular gasoline \\
Injected Fluid Temperature & $300 \mathrm{~K}$ \\
Vaporized Fluid Fraction & $30 \%$ \\
\hline
\end{tabular}

\subsubsection{Crank-Connecting Rod System Modeling}

The crank-connecting rod system is composed of crankcase, cranktrain, scavenging passage, and scavenging port on the cylinder wall. Moreover, crankcase and cranktrain are both core structures of this system. The former seals off the fresh charge of air-fuel mixture and subsequently delivers it into the cylinder through scavenging passage and scavenging port, and the latter converts the reciprocal rectilinear motion of piston into rotational motion and outputs shaft power. To model these two, the dedicated simulation modules EngCrankcase and EngCrankTrain are selected, respectively. The parameter settings are shown in Tables 8 and 9. It should be noted that the whole crankcase is divided into two "half-crankcases" as described above, and these two are labeled as "crankcase-1" and "crankcase-2", respectively.

Table 8. Parameter settings of the simulation module of crankcase.

\begin{tabular}{cc}
\hline Parameter & Value \\
\hline Crankcase Compression Ratio & 1.41 \\
Piston Skirt Temperature & $450 \mathrm{~K}$ \\
Cylinder Wall Temperature & $500 \mathrm{~K}$ \\
Crankcase Wall Temperature & $350 \mathrm{~K}$ \\
Heat Transfer Model & WoschniGT \\
\hline
\end{tabular}

Table 9. Parameter settings of the simulation module of cranktrain.

\begin{tabular}{cc}
\hline Parameter & Value \\
\hline Engine Type & 2-stroke \\
Speed or Load Specification & speed \\
Start of Cycle & $114{ }^{\circ} \mathrm{CA}$ Before TDC \\
Firing Interval & $0{ }^{\circ} \mathrm{CA}$ \\
Connecting Rod Length & $110 \mathrm{~mm}$ \\
Piston-to-Crank Offset & $0 \mathrm{~mm}$ \\
TDC Clearance Height & $0.4 \mathrm{~mm}$ \\
Friction Loss Model & EngFrctionCF \\
\hline
\end{tabular}


As displayed in Table 8, the crankcase compression ratio is set as 1.41. It should be noted that each crankcase part represents the chamber below each cylinder, so that the compression ratio should not be calculated using the crankcase volume of the entire engine. Instead, half of the entire crankcase volume is used for calculating the crankcase compression ratio for "crankcase-1" and "crankcase-2". Temperature parameters of piston skirt, cylinder wall, and crankcase wall are determined based on the 3D CFD calculation results and verified against the ground test data. In addition, the WoschniGT is selected as the heat transfer model of the crankcase simulation module [39-41]. With respect to the actual heat transfer process, the instantaneous heat transfer coefficient $h_{t r}$ is difficult to determine due to influence factors such as the physical property change, the variation of flow velocity, and the complicated phase transition. With bore $D_{c y l}$ and mean piston speed $v_{p m}$ recognized as geometric feature parameters in the Reynolds number $R e$, the WoschniGT proposes an empirical criterion to estimate the Nusselt number $N u$ as shown in Equation (6) [35]:

$$
N u=0.035 R e^{0.8}
$$

Based on the above criterion equation, the estimation equation for $h_{t r}$ is shown as follows [35]:

$$
h_{t r}=110 p_{c y l}^{0.8} \cdot T_{c y l}^{-0.53} \cdot D_{c y l}^{-0.2} \cdot\left[C_{f v} v_{p m}+C_{c c s} \cdot \frac{T_{c s} V_{s}}{p_{c s} V_{c s}\left(p_{c y l}-p_{m o}\right)}\right]
$$

where $p_{c y l}$ is cylinder pressure, $T_{c y l}$ is cylinder temperature, $C_{f v}$ is flow velocity coefficient, $C_{c c s}$ is the coefficient of combustion chamber shape, $T_{C S}$ is the temperature at the start of compression stroke, $p_{c S}$ is the cylinder pressure at the start of compression stroke, $V_{c S}$ is the cylinder volume at the start of compression stroke, $V_{s}$ is the displacement of a single cylinder, and $p_{m o}$ is the cylinder pressure under the motored condition.

As shown in Table 9, the engine type is defined as 2-stroke naturally. With respect to speed or load specification, speed is prescribed as the fixed parameter in a simulation case, and corresponding load variation will be calculated, which is also the most usual case. The start of the cycle is the crank angle before TDC at which calculation is started. Generally, this value should be equal to or after the scavenging port close, but before the start of combustion. Based on the intake and exhaust timing parameters displayed in Table 2, the start of cycle is determined as $114{ }^{\circ} \mathrm{CA}$ before TDC. Considering that two cylinders of the initial prototype engine are horizontally opposed, the firing interval of these two cylinders is set as $0{ }^{\circ} \mathrm{CA}$. Geometric structure parameters including connecting rod length, piston-to-crank offset, and TDC clearance height are also obtained by accurate measurement. Furthermore, the EngFrctionCF is selected as the friction loss model of cranktrain simulation module. The friction mean effective pressure (FMEP) is calculated through the following equation in this model [35]:

$$
\mathrm{FMEP}=\mathrm{IMEP}-\mathrm{BMEP}=\mathrm{FMEP}_{\mathrm{const}}+A_{F} \cdot p_{c y l, m a x}+B_{F} \cdot v_{p m}+C_{F} \cdot v_{p m}^{2}
$$

where IMEP is the indicated mean effective pressure, BMEP is the brake mean effective pressure, and FMEP ${ }_{\text {const }}$ is the constant pressure term of FMEP. The peak cylinder pressure factor $A_{F}$ represents the cylinder pressure dependence term in FMEP. $p_{c y l, m a x}$ is the maximum cylinder pressure. The mean piston speed factor $B_{F}$ represents the piston speed dependence term in FMEP, which has units of pressure/velocity. Equation (8) displays that $p_{c y l, m a x}$ (maximum cylinder pressure) and $v_{p m}$ (mean piston speed) are both important variables to calculate the FMEP. The former is extracted from experimental cylinder curves, while the latter is determined based on the real engine parameters including stroke and engine speed as shown in the following equation:

$$
v_{p m}=\frac{S \cdot n}{30} \times 10^{-3}
$$


where $S$ is stroke $(\mathrm{mm})$, and $n$ is engine speed $(\mathrm{r} / \mathrm{min})$.

Scavenging passage outside the cylinder linear is the only flow line to the cylinder. Similar to the simplification of intake pipe, the PipeRound simulation module is chosen to facilitate the modeling process. The scavenging passages connected to two cylinders are marked as "pipe-3(1)" and "pipe-3(2)", respectively. Because the scavenging passage is directly connected to the cylinder through the scavenging port, it should be noted that its wall temperature is set as $400 \mathrm{~K}$, while that of intake pipe is $300 \mathrm{~K}$.

Scavenging port is located at the end of scavenging passage. After the piston moves downward and uncovers the scavenging port, the fresh charge of air-fuel mixture is pumped into the cylinder through the scavenging port. When the scavenging port is closed again, the scavenging process also comes to an end. GT-ISE provides the specialized simulation module ValvePortConn to model intake/exhaust valve ports for 2-stroke engine. As shown in Figure 12, the variation curve of opening area with crank angle is required to be imported into the simulation module. With each module of crank-connecting system appropriately placed in GT-ISE, the layout is displayed in Figure 13.

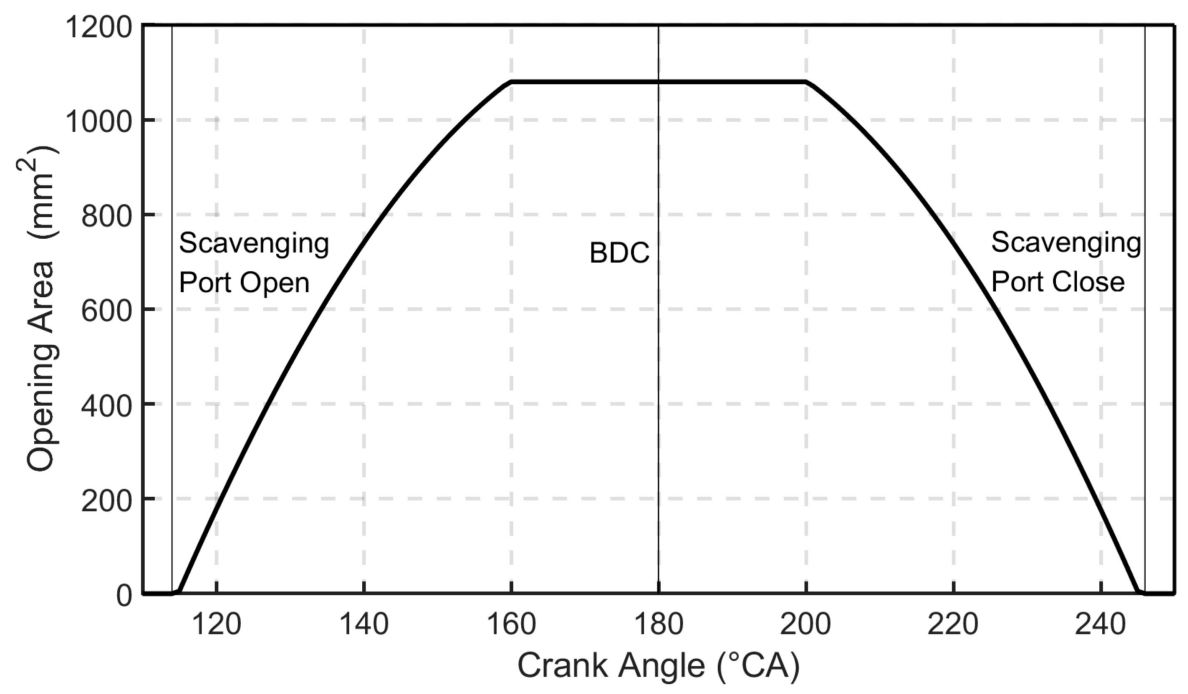

Figure 12. The variation curve of opening area with crank angle (scavenging port).

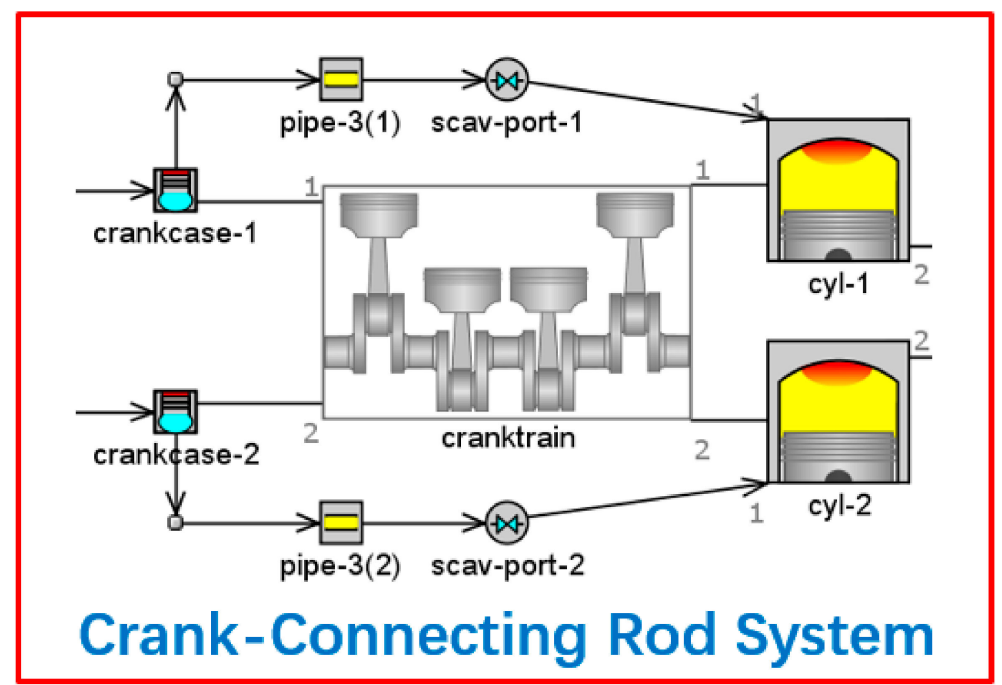

Figure 13. The layout of crank-connecting rod system in GT-ISE. 


\subsubsection{Cylinders Modeling}

With regard to the modeling of cylinders, GT-ISE also provides the dedicated simulation module EngCylinder. To distinguish these two horizontally opposed cylinders, "cyl-1" and "cyl-2" are marked respectively with Table 10 displaying the parameter settings. The first three parameters are extracted from Table 1. Head/bore area ratio and piston/bore area ratio are both calculated based on the accurate measurement results of relevant surface areas. With regard to temperature parameters of cylinder head, piston surface, and cylinder wall, these specific values are acquired in a way similar to those temperature parameters in Table 8.

Table 10. Parameter settings of the simulation module of cylinder.

\begin{tabular}{cc}
\hline Parameter & Value \\
\hline Stroke & $75 \mathrm{~mm}$ \\
Bore & $56 \mathrm{~mm}$ \\
Displacement of A Single Cylinder & $0.250 \mathrm{~L}$ \\
Head/Bore Area Ratio & 1.4 \\
Piston/bore Area Ratio & 1.1 \\
Cylinder Head Temperature & $550 \mathrm{~K}$ \\
Piston Surface Temperature & $550 \mathrm{~K}$ \\
Cylinder Wall Temperature & $500 \mathrm{~K}$ \\
Engine Combustion Model & EngCylCombSIWiebe \\
Engine Scavenging Model & EngCylScav \\
\hline
\end{tabular}

As shown in Table 10, the EngCylCombSIWiebe is selected as the engine combustion model. As a non-predictive combustion model, it imposes the burn rate for spark-ignition (SI) engines using a Wiebe function, which approximates the "typical" shape of an SI burn rate. The Wiebe equations are given below $[35,42,43]$ :

$$
\begin{gathered}
B S C=-\ln (1-B S) \\
B M C=-\ln (1-B M) \\
B E C=-\ln (1-B E) \\
S O C=A A-\frac{C D \cdot B M C^{1 /(W E+1)}}{B E C^{1 /(W E+1)}-B S C^{1 /(W E+1)}} \\
W C=\left[\frac{C D}{B E C^{1 /(W E+1)}-B S C^{1 /(W E+1)}}\right]^{-(W E+1)}
\end{gathered}
$$

where $A A$ is anchor angle (i.e., the number of crank angle degrees between TDC and typically the $50 \%$ combustion point of the Wiebe curve), $C D$ is the specified combustion duration which by default excludes the first $10 \%$ and last $10 \%$ of the total combustion duration, WE is Wiebe exponent, $B S$ is the burned fuel percentage at duration start (the default is $10 \%$ ), $B M$ is the burned fuel percentage at anchor angle (the default is $50 \%$ ), $B E$ is the burned fuel percentage at duration end (the default is $90 \%$ ), the relevant calculated constants include burned start constant BSC, burned midpoint constant $B M C$, burned end constant $B E C$, Wiebe constant $W C$, and the start of combustion $S O C$ which also has units of crank angle.

Derived from these corresponding Wiebe equations, cumulative burn rate Combustion $(\varphi)$ is calculated and normalized to 1.0 as shown in Equation (15), where $\varphi$ represents instantaneous crank angle and FFB represents the fraction of fuel burned [35,42,43]. Generally, the combustion starts at 0.0 ( $0 \%$ burned) and progresses to 1.0 (100\% burned):

$$
\text { Combustion }(\varphi)=\text { FFB } \cdot\left[1-e^{-W C \cdot(\varphi-S O C)^{W E+1}}\right]
$$


The intake and exhaust process of the initial prototype engine is completed with the help of scavenging flow supplied by the crankcase. The fresh charge of air-fuel mixture is guided through the scavenging port in purpose to sweep the burned gases out of the cylinder. It is in the cylinder that the scavenging process takes place. As a consequence, the EngCylScav is determined as the engine scavenging model with the scavenging profile displayed in Figure 14 as an indispensable model input [31].

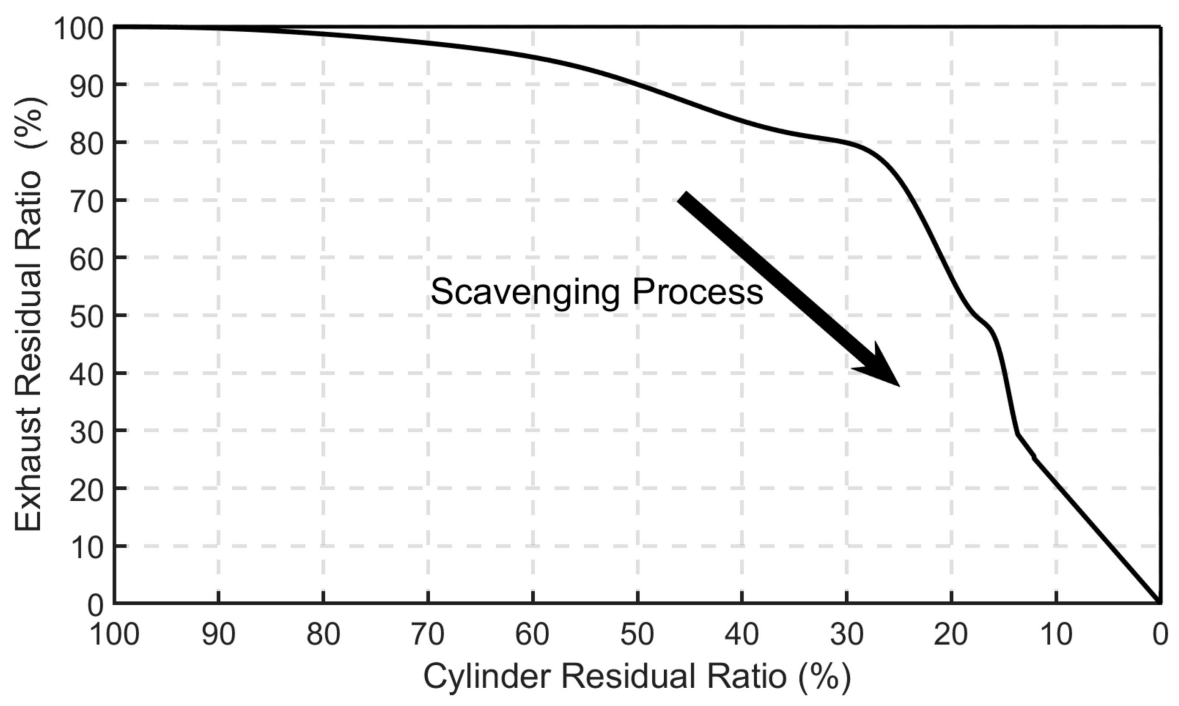

Figure 14. The scavenging profile.

As shown in Figure 14, the following two attributes establish the scavenging profile: the cylinder residual ratio (i.e., the mass fraction of burned gases in the cylinder) and the exhaust residual ratio (i.e., the mass fraction for burned gases in the exhaust pipe). The scavenging behavior in the cylinder can be visualized using Figure 15 below. The fresh air comes into the cylinder, displacing the burned gases. When the inlet first starts to bring in fresh air, the cylinder residual ratio and exhaust residual ratio will be 1.0. As the fresh air enters the cylinder, the cylinder residual ratio will decrease. However, in a realistic engine, the exhaust residual ratio will remain at or near 1.0 for some time, until the fresh charge mass increases enough to reach the outlet. Once the fresh charge has reached the outlet, it will exit, reducing the exhaust residual ratio. As time progresses, short-circuiting will reduce the exhaust residual ratio to a very low level.

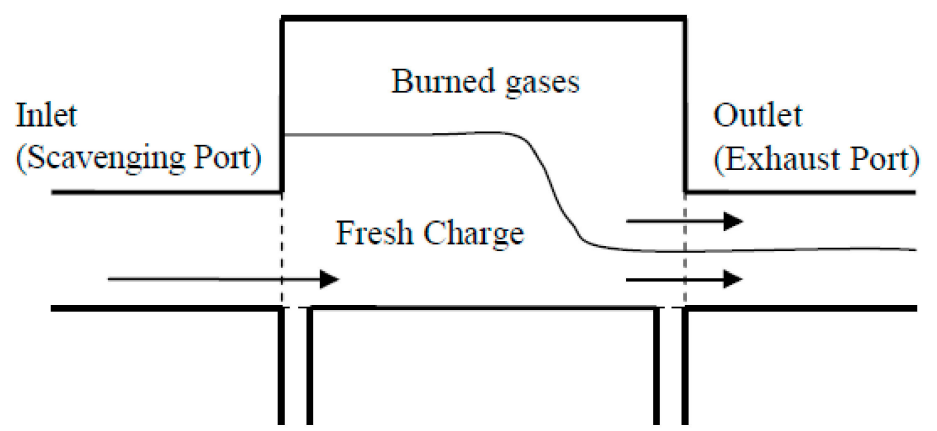

Figure 15. The scavenging behavior in the cylinder.

\subsubsection{Exhaust System Modeling}

The exhaust system comprises exhaust port, exhaust pipe, and atmospheric environment. In the expansion stroke, the piston begins to descend. Shortly afterward, the exhaust port is uncovered and 
the burned gases in the cylinder is discharged into the exhaust pipe through the exhaust port. Similar to the modeling process of scavenging port, the specialized simulation module ValvePortConn is selected to model the exhaust port. As shown in Figure 16, the variation curve of opening area with crank angle is imported into the simulation module. The exhaust ports on the two cylinders are marked with "exh-port-1" and "exh-port-2" respectively. Two exhaust pipes are modeled through the PipeRound simulation module. What makes it different is that its wall temperature is set as $800 \mathrm{~K}$, significantly exceeding that of intake pipe (300 K). In addition, these two exhaust pipes are labeled as "exh-pipe- 1 " and "exh-pipe-2" to distinguish them. It should be noted that the exhaust system must be fitted with sound-absorbing elements to eliminate the loud noise when the initial prototype engine is operated. The two dedicated Muffler simulation modules are selected to model this integral part in the exhaust system, and these two mufflers are labeled as "muffler-1" and "muffler-2" respectively. With respect to the atmospheric environment, it is all the same as that in the intake system. Correspondingly, the two EndEnvironment simulation modules used are named as "outlet-1" and "outlet-2". Finally, the layout of exhaust system in GT-ISE is shown in Figure 17.

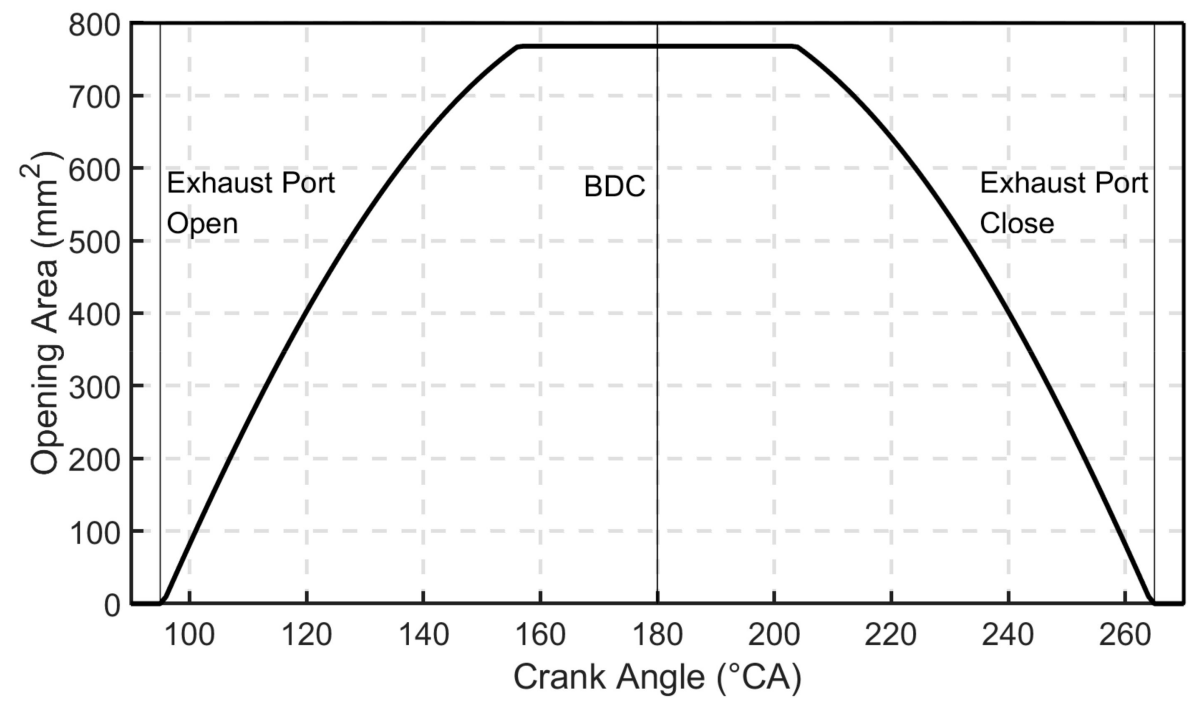

Figure 16. The variation curve of opening area with crank angle (exhaust port).

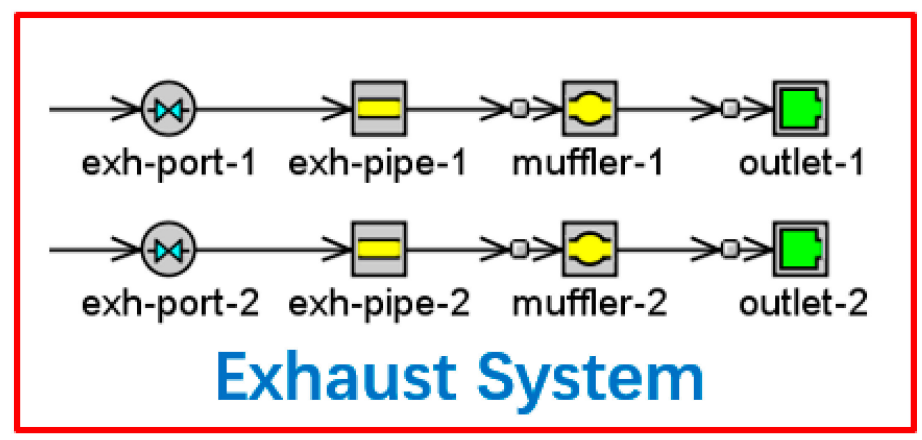

Figure 17. The layout of exhaust system in GT-ISE.

\subsubsection{Integrated Simulation Platform}

The cylinder pressure curve is one of the most important criteria for verifying the accuracy of the calculation results of the simulation platform of the initial prototype engine [31,32]. To enable real-time monitoring in the simulation process, the related cylinder pressure monitoring simulation modules are added into the established model. Two monitoring modules responsible for "cyl-1" and "cyl-2" are marked as "Monitor-1" and "Monitor-2", respectively. In addition, a single monitoring module includes a sensor simulation module SensorConn and an oscilloscope simulation module 
MonitorSignal. With the help of the monitoring module, the cylinder pressure data obtained from the current iterative cycle can be output and visualized in real time.

Despite some simplifications made in the modeling process, all of the above-mentioned systems are modeled appropriately. These submodels constitutes the integrated simulation platform of the initial prototype engine. With them properly connected to each other, the layout of the integrated simulation platform in GT-ISE can be shown in Figure 18.

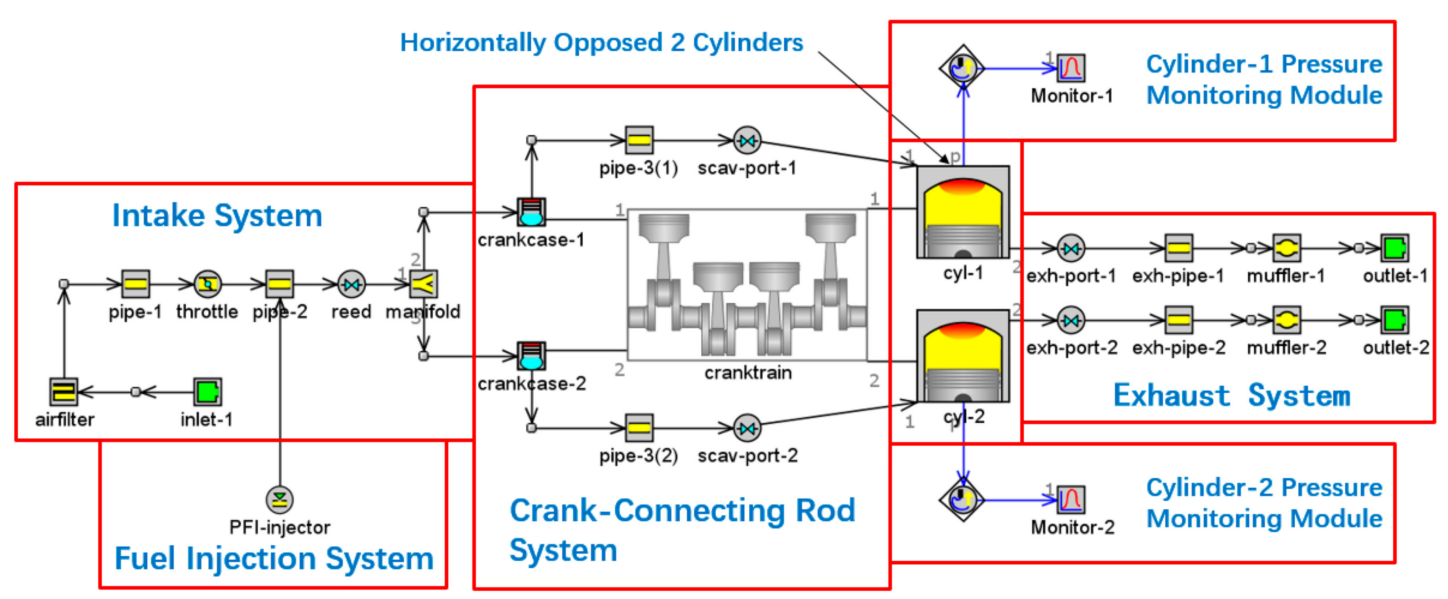

Figure 18. The layout of the integrated simulation platform in GT-ISE (initial prototype engine).

\subsection{Experiment Platform Construction of the Initial Prototype Engine}

To fulfill the requirement of subsequent comparative simulation, the accuracy of the calculation results of the simulation platform needs to be guaranteed. As a prerequisite, the crucial parameter configuration of the simulation platform should be calibrated against the test data before performing the simulation. Therefore, the experiment platform of the initial prototype engine is constructed to obtain both necessary and sufficient test data. As shown in Figure 19, a snapshot of the experiment platform is presented.

When the initial prototype engine is running, important performance evaluation indicators (i.e., engine speed, brake power, and brake torque) are measured by the AC dynamometer. Cylinder pressure signal is collected by the measuring spark plugs with miniature pressure sensors and processed by the charge amplifier. Associated with the crankshaft speed signal, cylinder pressure signal is transmitted into the combustion analyzer. Through the supporting software installed on the host PC, the acquired data can be visualized and analyzed in real time. Integrated with the air data collection module, the engine electronic control unit (ECU) outputs the control signal to the engine. For the initial prototype engine, what calls for special attention is fuel consumption rather than air consumption because the former directly reflects the fuel economy performance of engine, while the latter is generally not regarded as one of the core evaluation indicators. Therefore, the dynamic fuel mass flow meter is applied to measure the instantaneous fuel consumption, while the air flow meter is actually not in use. The fuel pressure regulator is located at the fuel return line to ensure that the fuel pressure is maintained at a constant level. In addition, two centrifugal fans are mounted toward the two cylinders of the initial prototype engine for the forced air cooling.

With respect to all this experimental equipment used to construct the platform, the accuracy of measurement instrument definitely has a significant impact on the precision of the collected test data. Table 11 displays the measurement accuracy specifications of above-mentioned main equipment as follows, where the measurement accuracy can be expressed as the relative ratio of measurement error to full scale range (FSR) in percentage. 


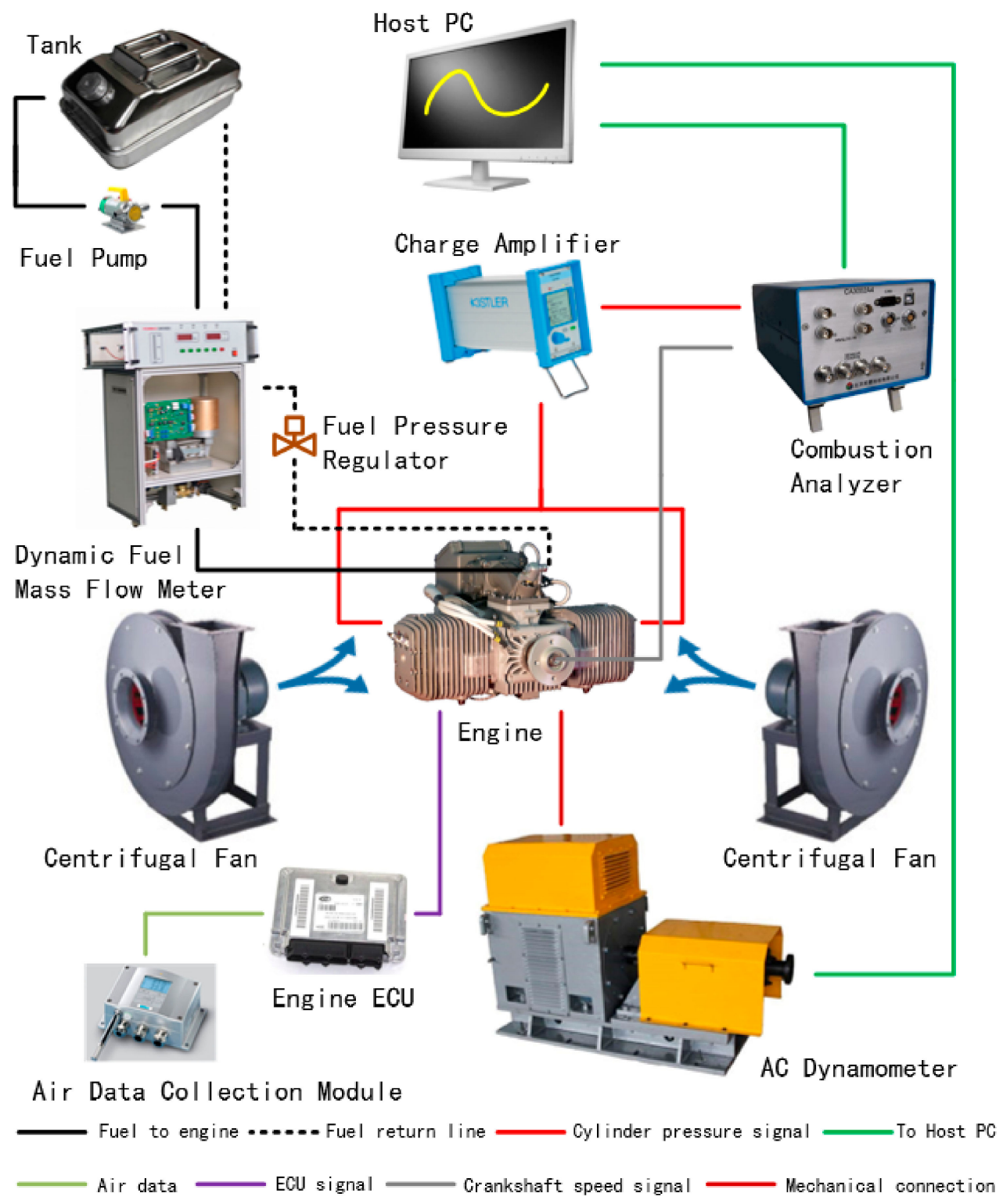

Figure 19. The experiment platform of the initial prototype engine.

Table 11. Measurement accuracy specifications of the main experimental equipment.

\begin{tabular}{cc}
\hline Experimental Equipment & Measurement Accuracy \\
\hline Dynamic fuel mass flow meter & $\pm 0.3 \%$ FSR \\
Charge amplifier & $\pm 0.3 \%$ FSR \\
Combustion analyzer & $\pm 0.5 \%$ FSR \\
AC dynamometer & $\pm 0.2 \%$ FSR \\
Air data collection module & $\pm 0.25 \%$ FSR \\
\hline
\end{tabular}

As mentioned in Section 1, the initial prototype engine is adopted as the power system for small and medium-sized UAVs. Hence, the engine operation conditions are determined based on the flight state of the UAV. The running status of the initial prototype engine is characterized by three typical operation conditions as listed in Table 12. When the UAV is taking off, full power output is needed for the UAV to climb to the cruise altitude as soon as possible. For this reason, the engine has to be operated under the full load condition. When the UAV reaches the cruise altitude, it will demand better engine fuel economy performance to extend its loitering time. Therefore, the engine needs to be 
operated under the medium load condition for lower fuel consumption. When the UAV begins to descend for landing, the corresponding requirements for power and engine speed are considerably reduced, thus engine is usually operated at the minimum load condition.

Table 12. Three typical operation conditions of the initial prototype engine.

\begin{tabular}{ccc}
\hline UAV Flight State & Operation Condition & Specification \\
\hline Taking off & full load & $\begin{array}{c}\text { throttle valve opening }=100 \% \\
\text { engine speed }=6500 \mathrm{r} / \mathrm{min} \\
\text { throttle valve opening }=60 \% \\
\text { engine speed }=5800 \mathrm{r} / \mathrm{min} \\
\text { throttle valve opening }=20 \% \\
\text { engine speed }=4500 \mathrm{r} / \mathrm{min}\end{array}$ \\
\hline
\end{tabular}

It should be noted that simulating the occurrence of knock/detonation is not the main function of GT-ISE. Additionally, obvious knock/detonation phenomenon was not observed when the initial prototype engine was actually operated. Therefore, little attention has been paid to that point in this paper.

\section{Simulation Platform Calibration of the Initial Prototype Engine}

During the modeling process of the simulation platform, the specific values of some important parameters are hard to determine because of the lack of correspondence to the engine specifications (structure dimensions, operation conditions, performance curves, etc.). Therefore, the recommended values provided by GT-ISE are adopted when preliminarily constructing the simulation platform, so that we are not sure whether the simulation results show good agreement with the real experimental results. In other words, we cannot guarantee whether the simulation platform is reliable and accurate enough. To address this problem, we construct the experimental platform, carry out a series of tests, and obtain necessary experimental results (cylinder curves, brake power, brake torque, BMEP, etc.). Based on the experimental results, we are able to calibrate some importance parameters in the pre-established simulation platform, which is also the main content of this section.

As mentioned above, the established simulation platform needs to be calibrated against the test data to lay a solid foundation for subsequent comparative simulation, which means these above parameters are required to be accurately reconfigured so that the simulation results can match well with the test data. The simulation platform calibration can be divided into four subtasks including the friction loss model calibration, the effective compression ratio calibration, the heat release curve calibration, and the discretization length calibration.

\subsection{Friction Loss Model Calibration}

Ordinarily, the engine friction loss has direct influence on the power evaluation indicators of the initial prototype engine. As shown in Equation (8), the EngFrctionCF model calculates the FMEP based on these vital coefficients including FMEP ${ }_{\text {const }}, A_{F}, B_{F}$, and $C_{F}$. Therefore, the specific values of above coefficients are fine-tuned in the recommended range. It is worthy of special mention that all of the three typical operation conditions in Table 12 are supposed to be taken into consideration. The final calibration results are shown in Table 13, where the relative error is calculated as follows:

$$
\text { Relative Error }=\left|\frac{\mathrm{SV}-\mathrm{EV}}{\mathrm{EV}}\right| \times 100 \%
$$

where SV is the simulation value calculated by the simulation platform, and EV is the experiment value obtained from the experiment platform. It can be observed that the friction model is calibrated against the obtained experimental data including brake power, brake torque, and BMEP. 
Table 13. Calibration results of friction loss model.

\begin{tabular}{|c|c|c|c|c|c|c|c|}
\hline \multirow{2}{*}{$\begin{array}{l}\text { Operation } \\
\text { Condition }\end{array}$} & \multicolumn{2}{|c|}{$\begin{array}{l}\text { Brake Power } \\
(\mathbf{k W})\end{array}$} & \multicolumn{2}{|c|}{$\begin{array}{c}\text { Brake Torque } \\
(\mathbf{N} \cdot \mathbf{m})\end{array}$} & \multicolumn{2}{|c|}{$\begin{array}{c}\text { BMEP } \\
\text { (bar) }\end{array}$} & \multirow[t]{2}{*}{ Relative Error } \\
\hline & SV & EV & SV & EV & SV & EV & \\
\hline minimum load & 11.10 & 10.92 & 23.55 & 23.16 & 2.96 & 2.91 & $1.68 \%$ \\
\hline medium load & 24.55 & 24.21 & 40.43 & 39.87 & 5.13 & 5.06 & $1.38 \%$ \\
\hline full load & 28.48 & 28.22 & 41.84 & 41.46 & 5.31 & 5.26 & $0.91 \%$ \\
\hline $\begin{array}{l}\text { Operation } \\
\text { Condition }\end{array}$ & \multicolumn{2}{|c|}{$\begin{array}{l}\text { FMEP }_{\text {const }} \\
\text { (bar) }\end{array}$} & \multicolumn{2}{|c|}{$A_{F}$} & \multicolumn{2}{|c|}{$\begin{array}{c}B_{F} \\
(\operatorname{bar} /(\mathrm{m} / \mathrm{s}))\end{array}$} & $\begin{array}{c}C_{F} \\
\left(\mathrm{bar} /(\mathrm{m} / \mathrm{s})^{2}\right)\end{array}$ \\
\hline minimum load & \multicolumn{2}{|c|}{0.27} & \multicolumn{2}{|c|}{0.0038} & \multicolumn{2}{|c|}{0.0021} & 0.0006 \\
\hline medium load & \multicolumn{2}{|c|}{0.31} & \multicolumn{2}{|c|}{0.0042} & \multicolumn{2}{|c|}{0.0029} & 0.0010 \\
\hline full load & \multicolumn{2}{|c|}{0.32} & \multicolumn{2}{|c|}{0.0045} & \multicolumn{2}{|c|}{0.0035} & 0.0012 \\
\hline
\end{tabular}

\subsection{Effective Compression Ratio Calibration}

Compression ratio is a tremendously important structural parameter for the initial prototype engine. It should be noted that the value shown in Table 1 is actually geometric compression ratio $\varepsilon_{\text {geo }}$, which is a theoretical value. In addition, it is determined by geometric dimensions including bore $D_{c y l}$, stroke $S$, and the volume of combustion chamber on the cylinder head $V_{c c}$. The related calculating formula is given below:

$$
\varepsilon_{\text {geo }}=1+\frac{\pi D_{c y l}^{2} S}{4 V_{c c}}
$$

However, the engine compression ratio will fall below the theoretical compression ratio when the engine is actually running. Generally, trapped compression ratio is far more relevant to ported 2-stroke engines than geometric compression ratio. Figure 20 displays a sectional drawing of the engine cylinder, where $h_{s c}$ represents the distance from TDC surface to scavenging ports, $l_{s c}$ is the length of scavenging ports, $h_{e x}$ represents the distance from TDC surface to exhaust port, and $l_{e x}$ is the length of exhaust port. It can be seen that, when the piston goes from BDC to TDC, the effective compression stroke is definitely lower than the complete engine stroke. Therefore, it causes the "real compression ratio" (i.e., effective compression ratio) to be definitely lower than the theoretical compression ratio, and this should be taken into account when setting the related parameter in the cylinder simulation module.

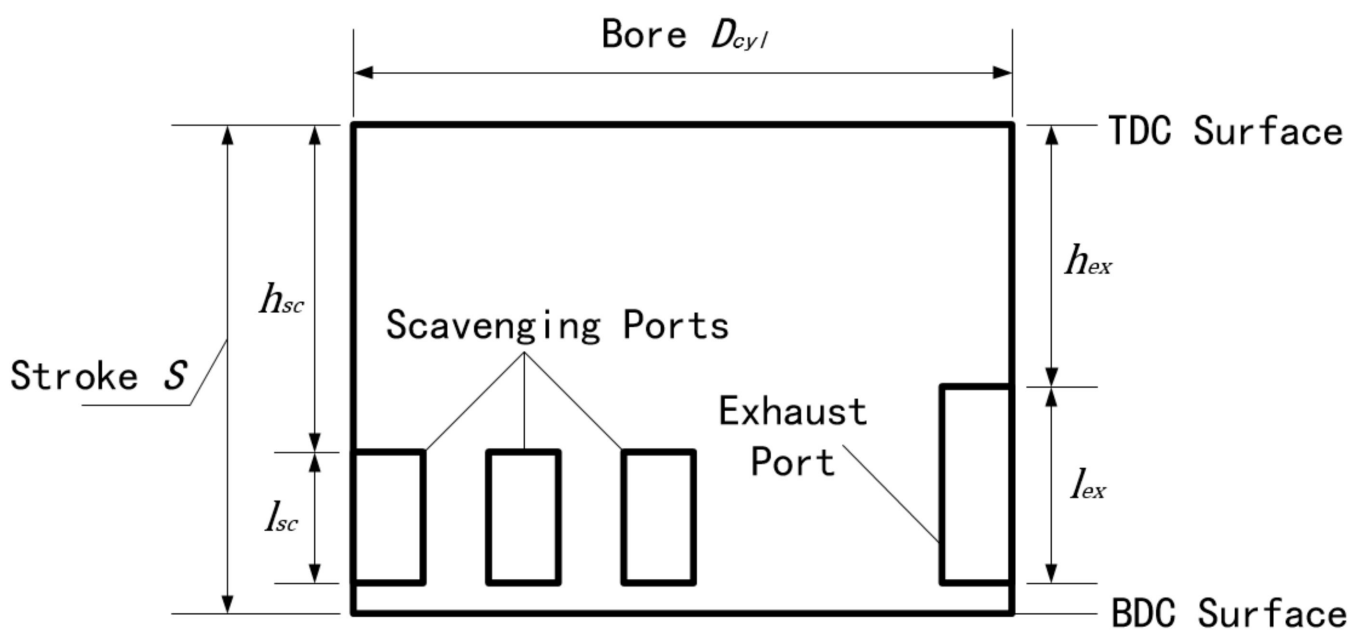

Figure 20. A sectional drawing of the engine cylinder.

Similarly, three typical operation conditions are calibrated against the test data. Considering that the compression ratio has the most significant effect on the cylinder pressure, the relevant data points obtained from the actual engine running are selected as the benchmark. With respect to the 
minimum load operation condition, Figure 21 displays the effect of different compression ratios on the cylinder curve calculated by simulation platform. In addition, experimental data points collected during the real operation are also displayed in Figure 21. Best agreement between the simulation curve and the experimental data points can be observed when the compression ratio is set to 9.2. The same conclusion can be drawn with regard to the other two operation conditions. Because of the limited space, these two figures are presented in Appendix A. From a comprehensive perspective of all the three typical operation conditions, the effective compression ratio is finally set to 9.2.

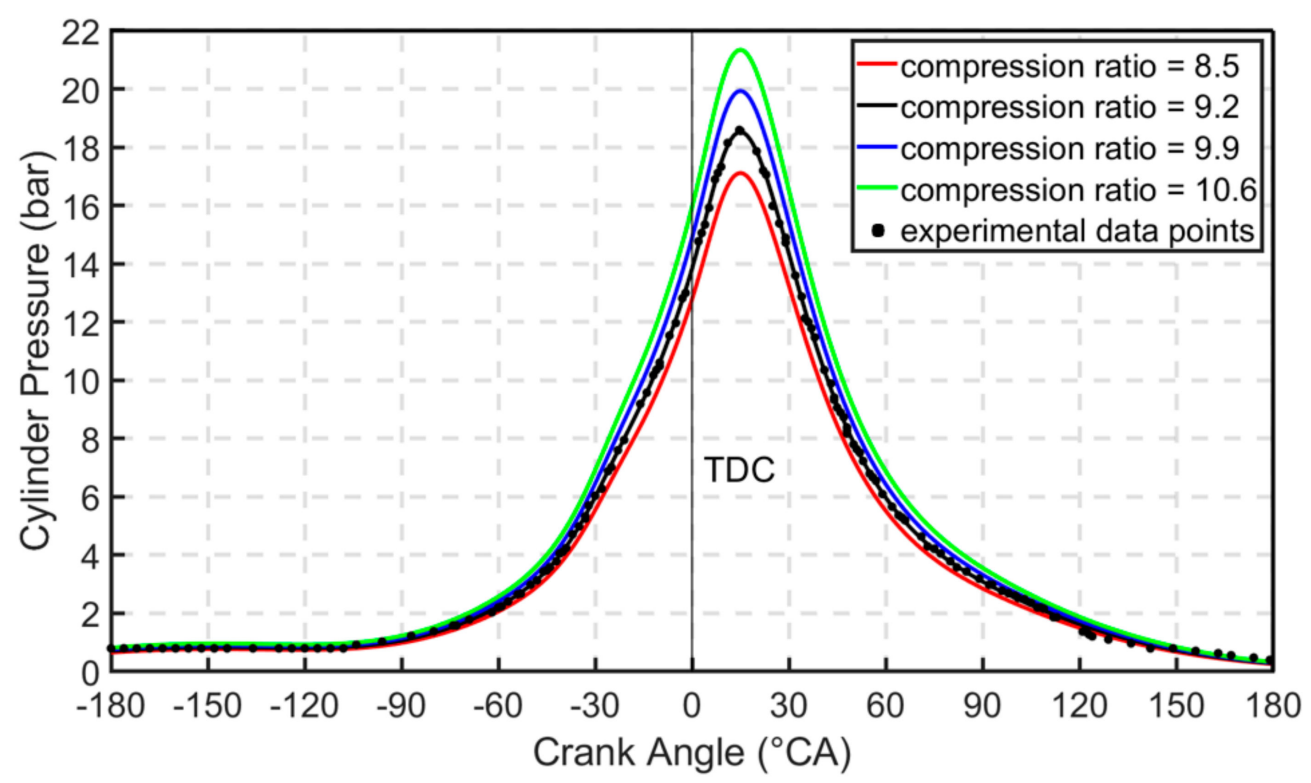

Figure 21. Comparison between cylinder curves with different compression ratios (minimum load).

\subsection{Heat Release Rate Curve Calibration}

As displayed in Table 10, the EngCylCombSIWiebe is selected as the engine combustion model. Derived from Equations (10)-(15), the cumulative burn rate Combustion $(\varphi)$ is calculated and applied to describe the combustion process in the cylinder. According to these Wiebe equations, the accurate values of $A A, C D$, and $W E$ are needed to be specified to calculate Combustion $(\varphi)$. Considering the complete engine combustion process, $\operatorname{Combustion}(\varphi)$ is the ratio of the integration of heat release rate on crank angle to the total heat release, as shown in the following equations:

$$
\begin{aligned}
Q_{\text {all }} & =\int_{S O C}^{E O C} d Q_{b} \\
\operatorname{Combustion}(\varphi) & =\frac{1}{Q_{\text {all }}} \cdot \int_{S O C}^{\varphi} d Q_{b} \times 100 \%
\end{aligned}
$$

where $S O C$ is the start of combustion which has units of crank angle, EOC is the end of combustion which also has units of crank angle, $Q_{\text {all }}$ is the total heat release from $S O C$ to $E O C$, and $d Q_{b}$ is heat release rate. Apparently, the heat release rate curve features prominently in the calculation of $\operatorname{Combustion}(\varphi)$, and Equation (20) gives the calculating formula of instantaneous heat release rate [39]:

$$
\frac{d Q_{b}}{d \varphi}=\left[\frac{1}{\kappa-1}\left(V_{c c} \cdot \frac{d p_{c y l}}{d \varphi}+\kappa p_{c y l} \cdot \frac{d V_{c c}}{d \varphi}\right)-\frac{p_{c y l} V_{c c}}{(\kappa-1)^{2}} \cdot \frac{d \kappa}{d \varphi}\right]+\alpha_{c c} F_{c c}\left(T_{c y l}-T_{c c}\right)
$$

where $\alpha_{c c}$ is the heat transfer coefficient of combustion chamber wall, $F_{c c}$ is the heat transfer area of combustion chamber wall, $T_{c c}$ is the temperature of combustion chamber wall. With regard to $T_{c c}$, there exists slight fluctuations in the actual working cycles, but generally it is deemed as a fixed value. 
Similarly, the isentropic exponent of working substance $\kappa$ is usually set to a constant value in the permissive range (1.2-1.34) for the ease of calculation [37].

Based on the structural parameters of the initial prototype engine and the test data of cylinder pressure, the experimental curves of heat release rate can be obtained. Similar to the calibration of friction loss model, crucial model parameters including $A A, C D$, and $W E$ are supposed to be fine-tuned within the appropriate range to make sure that the heat release curves calculated by the simulation platform match well with the corresponding experimental curves. Considering all of the three typical operation conditions, the final calibration results are provided in Table 14. Taking the minimum load operation condition as an example, the comparison between experimental curve and simulation curve is shown in Figure 22 and it can be noticed that the simulation results offer a good fit for the experimental curve. Limited by the space, figures of the other two operation conditions are presented in Appendix A.

Table 14. Parameter settings of the engine combustion model.

\begin{tabular}{ccccc}
\hline $\begin{array}{c}\text { Operation } \\
\text { Condition }\end{array}$ & $\begin{array}{c}\text { Anchor Angle } \\
\boldsymbol{A A}\end{array}$ & $\begin{array}{c}\text { Combustion Duration } \\
\boldsymbol{C D}\end{array}$ & $\begin{array}{c}\text { Wiebe Exponent } \\
\text { WE }\end{array}$ & $\begin{array}{c}\text { Start of Combustion } \\
\text { SOC }\end{array}$ \\
\hline Lull Load & $8.5^{\circ} \mathrm{CA}$ After TDC & $32.5^{\circ} \mathrm{CA}$ & 2.00 & $25.4^{\circ} \mathrm{CA}$ Before TDC \\
Medium Load & $8.5^{\circ} \mathrm{CA}$ After TDC & $32.0^{\circ} \mathrm{CA}$ & 1.98 & $24.6^{\circ} \mathrm{CA}$ Before TDC \\
Minimum Load & $10.5^{\circ} \mathrm{CA}$ After TDC & $25.5^{\circ} \mathrm{CA}$ & 1.92 & $15.4^{\circ} \mathrm{CA}$ Before TDC \\
\hline
\end{tabular}

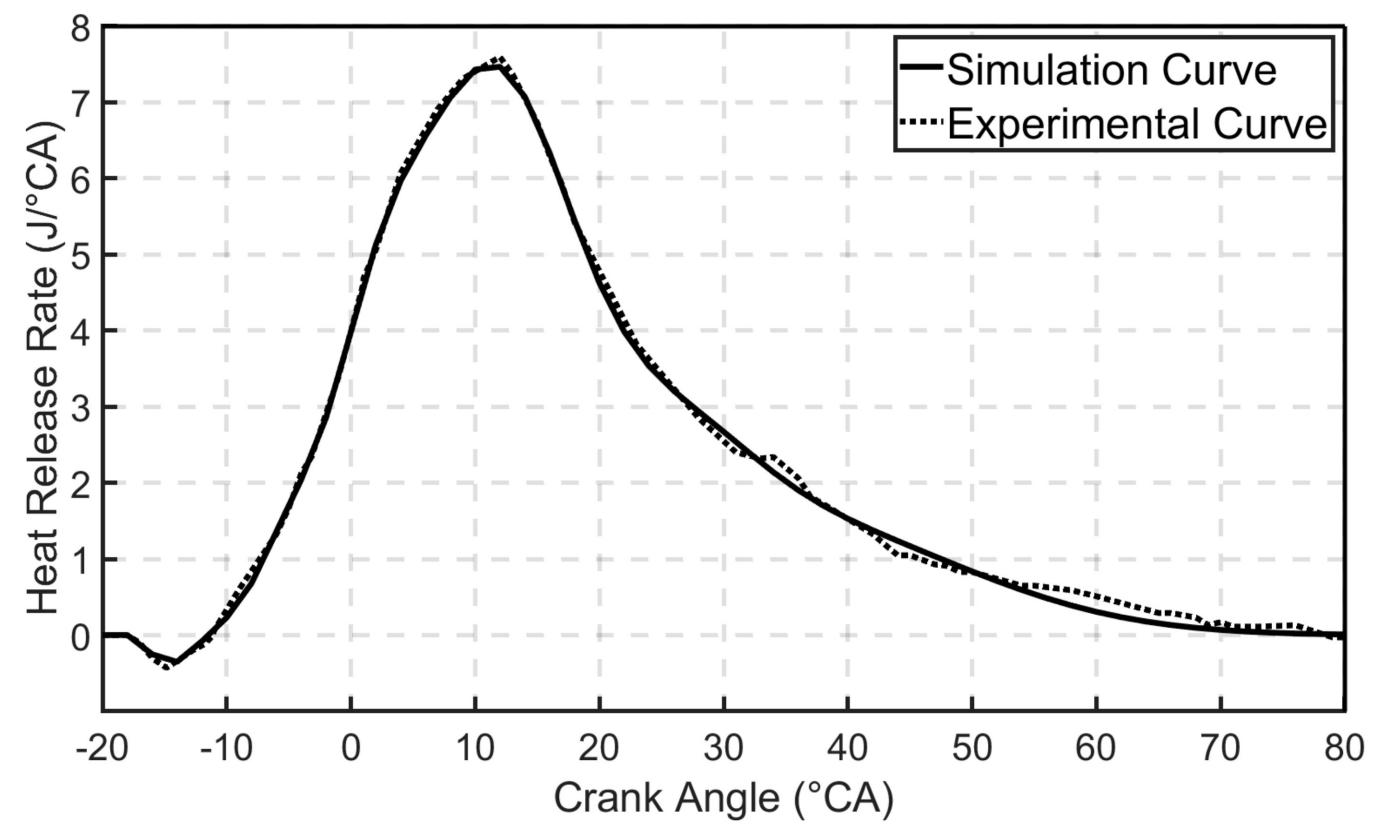

Figure 22. Comparison between heat release curves (minimum load).

\subsection{Discretization Length Calibration}

As described in Section 3.1, there are lots of pipe simulation modules in the simulation platform such as intake pipe, scavenging passage, and exhaust pipe. It should be noted that the flow fields in these pipes are actually continuous areas of control volume, but there are discretized to perform numerical calculation. Taking "pipe-2" as an example, discretization length should be input into its simulation module PipeRound. Considering that the actual length of "pipe-2" is $100 \mathrm{~mm}$, this section of intake pipe will be subdivided into 20 segments if the discretization length is set to $5 \mathrm{~mm}(100 \mathrm{~mm}=5 \mathrm{~mm} \times 20)$. Each one of these segments represents a $5 \mathrm{~mm}$ long section of "pipe-2". Theoretically, the shorter the discretization length, the finer the division of the corresponding pipe, the closer the discretized area is to the real continuous area of flow field, and the better the agreement of the simulation results with the real operation status of the initial prototype engine. 
However, the shorter the discretization length, the more the number of discrete nodes, the higher the dimensionality of the resulting set of algebraic equations required to be solved, and the longer the simulation time of one case. Therefore, there exists a trade-off relationship between simulation precision and time expenditure. Considering that tremendous simulation cases representing different operation conditions are required to be implemented, the total time cost will be extremely high if a single simulation case takes too long. Consequently, the specific value of discretization length needs to be calibrated to achieve a good balance between the simulation time of a single case and the accuracy of simulation results.

Using the full load operation condition as an example, the cylinder pressure curves with different discretization lengths are compared in Figure 23. As shown in Table 15, it takes $2254 \mathrm{~s}$ to finish a single simulation case when discretization length is set as $0.1 \mathrm{~mm}$. It has to be admitted that $2254 \mathrm{~s}$ or $37.5 \mathrm{~min}$ is not "too long", but there is not only one simulation case that needs to be calculated. Actually, there are dozens of simulation cases. Considering that, the simulation time of a single case should also be limited. Combined with the simulation time results given in Table 15, a conclusion that can be drawn is that the simulation time is still within an acceptable range while the cylinder pressure results are accurate enough when discretization length is set as $2.5 \mathrm{~mm}$. Therefore, $2.5 \mathrm{~mm}$ is finally selected as a reasonable discretization length.

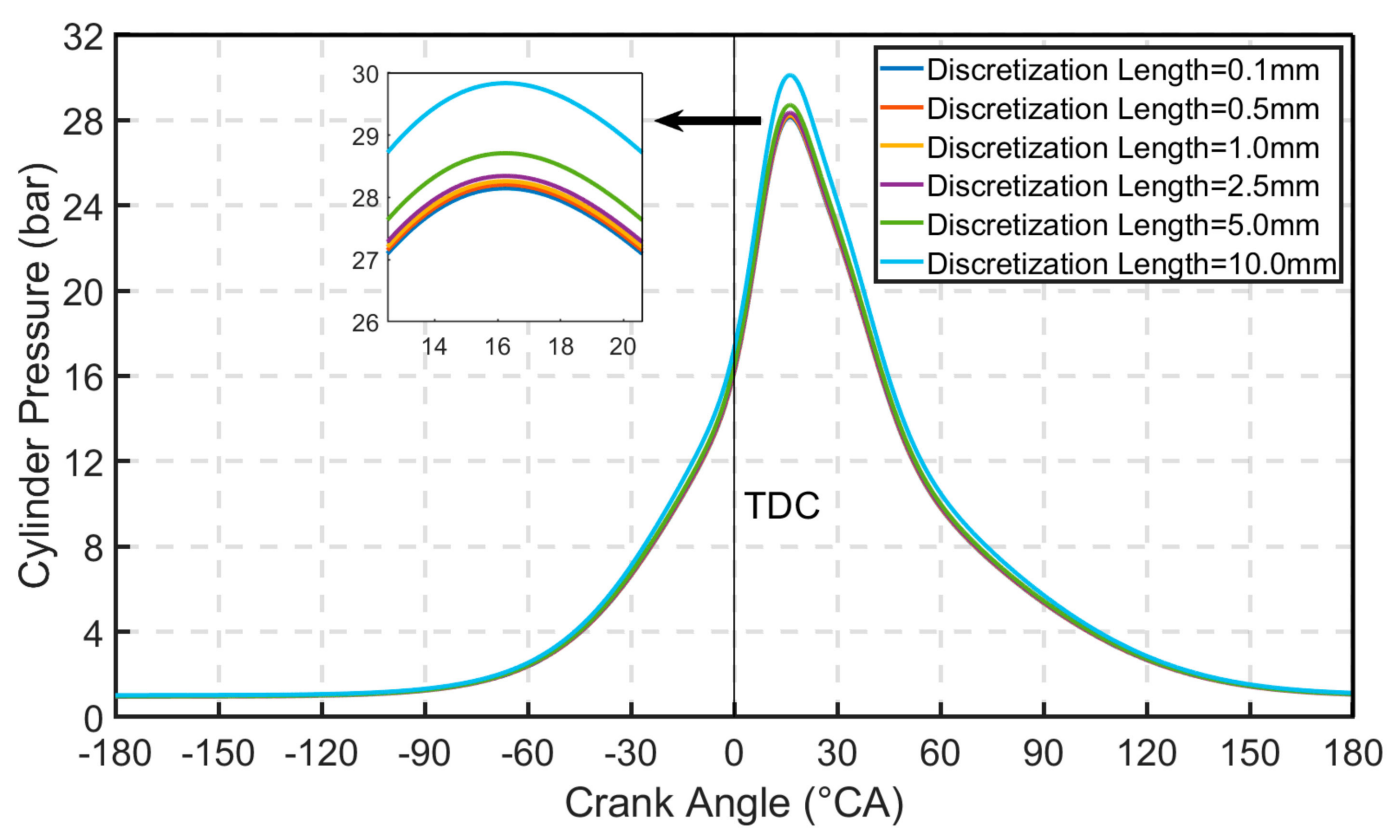

Figure 23. Comparison between cylinder curves with different discretization lengths (full load).

Table 15. The simulation time of a single case with different discretization lengths.

\begin{tabular}{cc}
\hline Discretization Length & Simulation Time (A Single Case) \\
\hline $0.1 \mathrm{~mm}$ & $2254 \mathrm{~s}$ \\
$0.5 \mathrm{~mm}$ & $713 \mathrm{~s}$ \\
$1.0 \mathrm{~mm}$ & $259 \mathrm{~s}$ \\
$2.5 \mathrm{~mm}$ & $102 \mathrm{~s}$ \\
$5.0 \mathrm{~mm}$ & $84 \mathrm{~s}$ \\
$10.0 \mathrm{~mm}$ & $69 \mathrm{~s}$ \\
\hline
\end{tabular}




\section{Simulation Platform Construction of the Reference Engine and the Retrofitted Engine}

\subsection{Simulation Platform Construction of the Reference Engine}

As mentioned in Section 2, the primary difference between the initial prototype engine and the reference engine is the fuel injection system. On the basis of the calibrated simulation platform of the initial prototype engine, the simulation platform of the reference engine with the CWI system can be constructed by making corresponding modifications.

With regard to the reference engine, the connecting orifice plays an important role in connecting the cylinder and the CWI tube as described in Section 2.2. The simulation module ValvePortConn is selected to model the connecting orifice. Similarly, the variation curve of opening area with crank angle is imported into this simulation module as shown in Figure 24. Connecting orifices on the two cylinder walls are marked as "orifice-1" and "orifice-2", respectively.

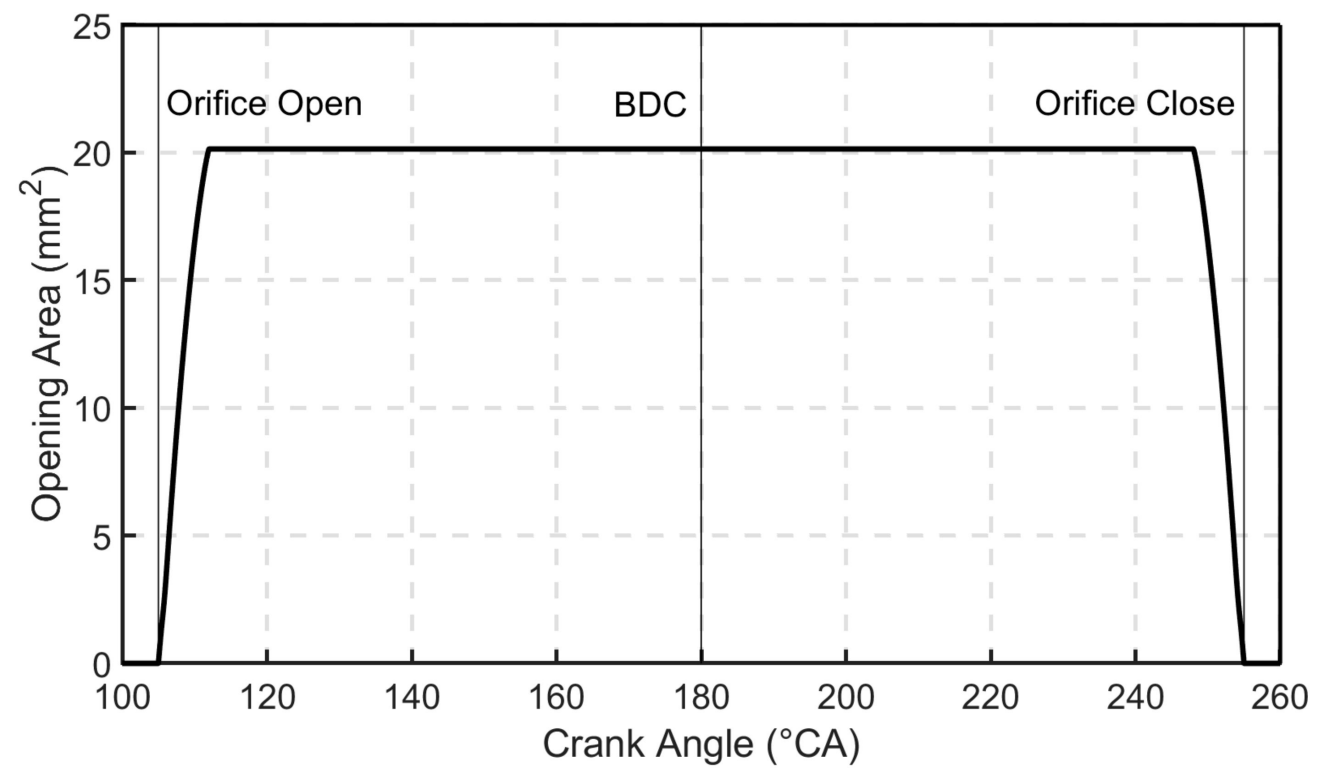

Figure 24. The variation curve of opening area with crank angle (connecting orifice).

In the CWI system, the CWI tube is a close-end round tube. Therefore, the PipeRound simulation module can be applied to model the CWI tube combined with the EndFlowCap simulation module. With respect to parameter settings, the wall temperature of the PipeRound simulation module is set to $450 \mathrm{~K}$ (that of scavenging passage is $400 \mathrm{~K}$ ) considering that a small proportion of burned gases flow into the CWI tube and thus heat the CWI tube. The CWI tubes for two cylinders are labeled as "CWI-tube-1" and "CWI-tube-2", respectively. As for the EndFlowCap simulation module, this component is use to cap the end of a pipe in order to prevent any flow through it, hence there is no input parameters needed.

As described in Section 3.1.2, the comparatively simple InjAF-RatioConn simulation module is chosen to model the conventional PFI system of the initial prototype engine. Different from the PFI system, the CWI system is more sensitive to injection timing and injection duration, and its injector needed to be modeled by a more suitable simulation module. The InjAFSeqConn module is chosen to describe the operation of sequential pulse fuel injector in the CWI system. By imposing the air-fuel ratio, the resultant injection duration which has units of crank angle $\left({ }^{\circ} \mathrm{CA}\right)$ is calculated for each injection event as follows [35]:

$$
\text { Injection Duration }=\frac{\eta_{v} \rho_{r e f} V_{s} n}{A / F} \cdot \frac{6}{\dot{m}_{i n j}}
$$


where $\eta_{V}$ is volumetric efficiency, $\rho_{r e f}$ is the reference density needed for calculating $\eta_{V}, V_{s}$ is the displacement of a single cylinder, $n$ is engine speed, $A / F$ is the imposed air-fuel ratio, and $\dot{m}_{i n j}$ is the delivery rate of injector. Similar to the InjAF-RatioConn simulation module mentioned in Section 3.1.2, air-fuel ratio is set as a variable parameter so that it can be adjusted to change the fuel injection quantity under different simulation conditions.

What calls for special attention is that other subsystems (intake system, crank-connecting rod system, cylinders, exhaust system, etc.) of the reference engine are the same as those of the initial prototype engine. Finally, the integrated simulation platform of the reference engine is constructed based on that of the initial prototype engine, and its layout is shown in Figure 25.

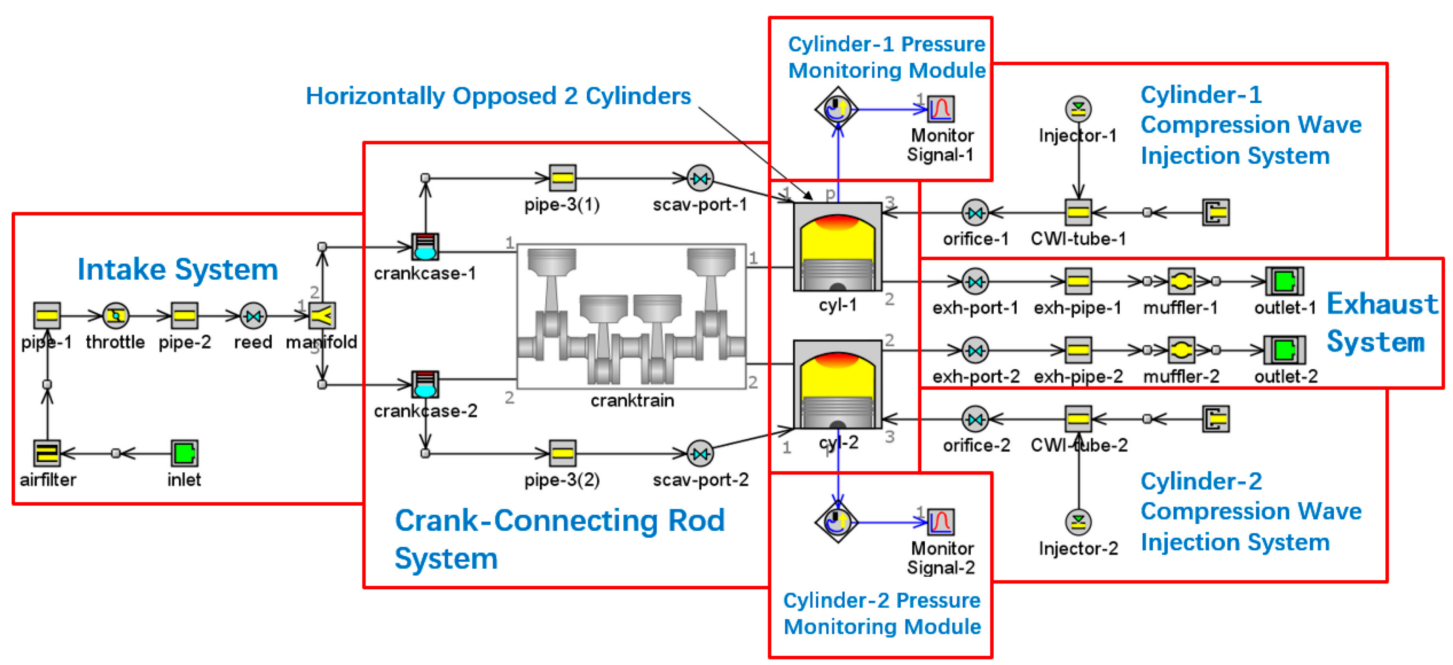

Figure 25. The layout of integrated simulation platform in GT-ISE (reference engine).

\subsection{Simulation Platform Construction of the Retrofitted Engine}

Similar to the reference engine, the major difference between the initial prototype engine and the retrofitted engine is the fuel injection system. Based on the calibrated simulation platform of the initial prototype engine, the simulation platform of the retrofitted engine can be set up by making corresponding modifications about the SPSDI system.

As described in Section 2.3, the resonance tube in the SPSDI system plays a similar role to the CWI tube in the CWI system. Therefore, the PipeRound and EndFlowCap simulation modules are also used to model the resonance tube. For the retrofitted engine, there is no connecting orifice on the cylinder wall and the resonance tube is not directly connected to the cylinder. Because there is no in-cylinder burned gases flowing into the resonance tube, its wall temperature is set to $400 \mathrm{~K}$ which is the same as that of scavenging passage $(400 \mathrm{~K})$ and lower than that of CWI tube $(450 \mathrm{~K})$. The resonance tubes for two cylinders are labeled as "res-tube-1" and "res-tube-2", respectively.

It is worthy of special mention that the resonance tube is connected to the end of scavenging passage at its open end. To connect these two ends and the scavenging port, a structure similar to T-branch pipe is added here, and the FlowSplitGeneral simulation module is selected to model it. For the two SPSDI systems, these two connecting structures are marked as "connect-1" and "connect-2", respectively.

The injector used in the SPSDI system is the same as that of the CWI system. The InjAFSeqConn simulation module is adopted to ensure flexible adjustment of injection timing and injection duration.

Similar to the reference engine, other subsystems of the retrofitted engine are the same as those of the initial prototype engine, which means that the integrated simulation platform of the retrofitted engine can also be established based on that of the initial prototype engine. The layout is shown in Figure 26. 


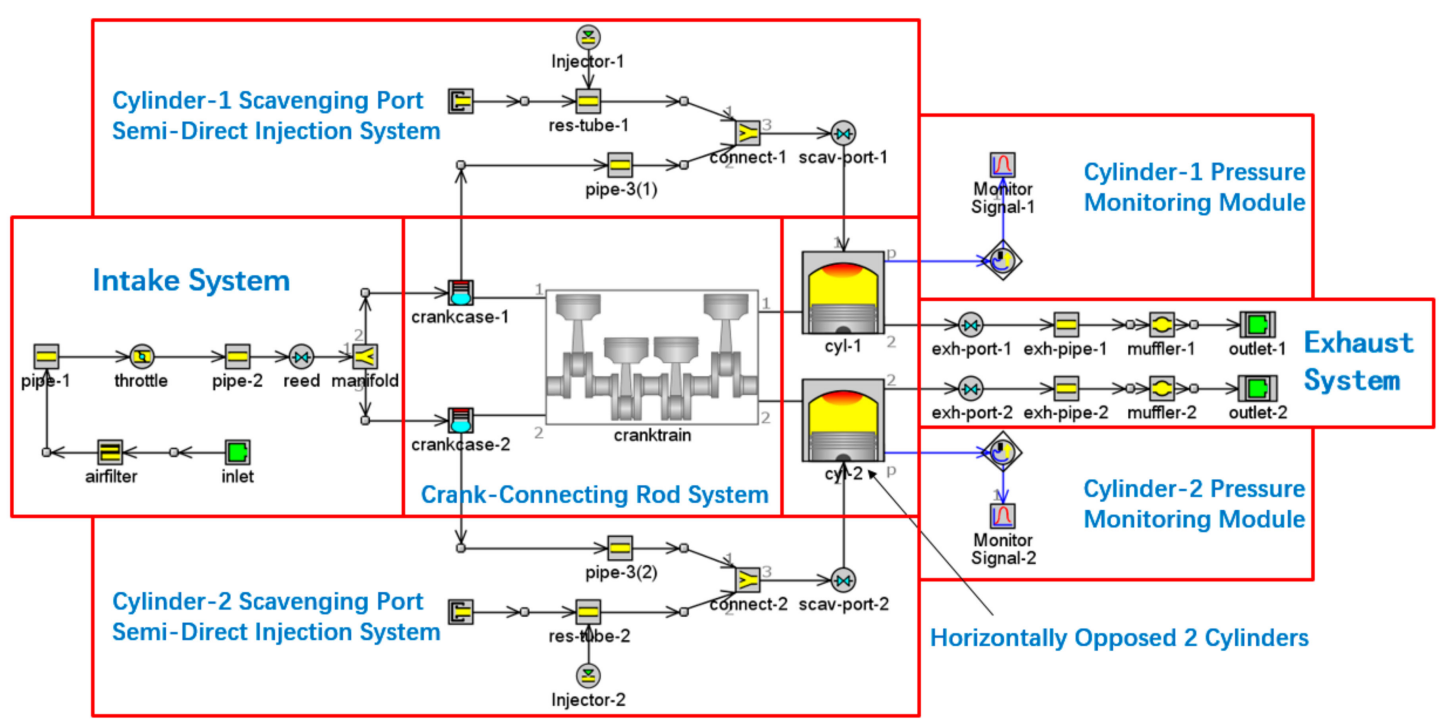

Figure 26. The layout of the integrated simulation platform in GT-ISE (retrofitted engine).

\section{Simulation Results Analysis}

Based on the pre-established simulation platforms of the above-mentioned three types of engines, the corresponding comparative simulation is performed with comprehensive simulation results shown in the related tables. Apart from power and fuel economy performance evaluation indictors including brake power, brake torque, IMEP, BMEP, ISFC, and BSFC, the cylinder curve of each simulation case is also calculated. Both the peak pressure and the crank angle at the peak pressure are extracted from the cylinder curve results to reflect the engine combustion characteristics.

\subsection{Performance Comparison of the Initial Prototype Engine}

First of all, the comparison of performance characteristics of the initial prototype engine supplied with regular gasoline/heavy fuel is conducted. It should be emphasized that the regular gasoline used here is 95 octane gasoline, and the heavy fuel used here is RP-3 aviation kerosene; comparison of the properties of these two different kinds of fuel is shown in Table 16 [14].

Table 16. Comparison of the properties of two kinds of fuel.

\begin{tabular}{ccc}
\hline Property & 95 Octane Gasoline & RP-3 Aviation Kerosene \\
\hline Molecular Weight & 113 & 141 \\
Flash point $\left({ }^{\circ} \mathrm{C}\right)$ & $-45-25$ & $35-51$ \\
Density $(\mathrm{kg} / \mathrm{L})$ & $0.70-0.75$ & $0.73-0.82$ \\
Kinematic viscosity at $20{ }^{\circ} \mathrm{C}\left(\mathrm{mm}^{2} / \mathrm{s}\right)$ & 0.80 & 1.25 \\
Spontaneous ignition temperature $\left({ }^{\circ} \mathrm{C}\right)$ & $510-530$ & 275 \\
Stoichiometric air-fuel ratio & 14.70 & 14.65 \\
Low heat value $(\mathrm{kg} / \mathrm{kJ})$ & 44.07 & 43.35 \\
\hline
\end{tabular}

Both speed and load characteristics are studied to fully explore the engine operation conditions. In the speed characteristics simulation cases, the throttle valve opening is set to $100 \%$ while the engine speed varies from $3000 \mathrm{r} / \mathrm{min}$ to $6500 \mathrm{r} / \mathrm{min}$. As for the load characteristics' simulation cases, the engine speed is set to the rated engine speed $6500 \mathrm{r} / \mathrm{min}$, while the throttle valve opening ranges from $10 \%-100 \%$. The specific values of these performance indicators are automatically calculated by the solver in GT-ISE. The comparative simulation results of engine speed characteristics and engine load characteristics are shown in Tables 17-20, respectively. RG refers to regular gasoline and HF refers to heavy fuel in the following tables. 
Table 17. Simulation results of engine speed characteristics (throttle valve opening $=100 \%$ ).

\begin{tabular}{ccccccccc}
\hline \multirow{2}{*}{ Engine Speed } & \multicolumn{2}{c}{$\begin{array}{c}\text { Brake Power } \\
(\mathbf{k W})\end{array}$} & \multicolumn{2}{c}{$\begin{array}{c}\text { Brake Torque } \\
\mathbf{( N \cdot m )}\end{array}$} & \multicolumn{2}{c}{$\begin{array}{c}\text { IMEP } \\
\text { (bar) }\end{array}$} & \multicolumn{2}{c}{$\begin{array}{c}\text { BMEP } \\
\text { (bar) }\end{array}$} \\
\cline { 2 - 10 } & HF & RG & HF & RG & HF & RG & HF & RG \\
\hline 6500 r/min & 24.10 & 28.48 & 35.41 & 41.84 & 5.20 & 5.74 & 4.49 & 5.31 \\
6000 r/min & 23.35 & 27.34 & 37.16 & 43.52 & 5.45 & 5.98 & 4.72 & 5.53 \\
5500 r/min & 22.28 & 25.87 & 38.67 & 44.91 & 5.62 & 6.14 & 4.91 & 5.70 \\
5000 r/min & 19.02 & 22.21 & 36.33 & 42.42 & 5.28 & 5.78 & 4.62 & 5.33 \\
4500 r/min & 16.68 & 19.68 & 35.39 & 41.76 & 5.16 & 5.61 & 4.50 & 5.16 \\
4000 r/min & 14.31 & 16.80 & 34.17 & 40.10 & 4.99 & 5.35 & 4.34 & 4.96 \\
3500 r/min & 12.22 & 14.65 & 33.34 & 39.96 & 4.73 & 5.18 & 4.24 & 4.84 \\
3000 r/min & 10.11 & 12.21 & 32.18 & 38.88 & 4.59 & 4.92 & 4.09 & 4.63 \\
\hline
\end{tabular}

Table 18. Simulation results of engine speed characteristics (throttle valve opening $=100 \%$ ).

\begin{tabular}{|c|c|c|c|c|c|c|c|c|}
\hline \multirow[t]{2}{*}{ Engine Speed } & \multicolumn{2}{|c|}{$\begin{array}{c}\text { ISFC } \\
(\mathrm{g} / \mathrm{kW} \cdot \mathrm{h})\end{array}$} & \multicolumn{2}{|c|}{$\begin{array}{c}\text { BSFC } \\
(\mathrm{g} / \mathrm{kW} \cdot \mathrm{h})\end{array}$} & \multicolumn{2}{|c|}{$\begin{array}{l}\text { Peak Pressure } \\
\text { (bar) }\end{array}$} & \multicolumn{2}{|c|}{$\begin{array}{l}\text { Crank Angle at the } \\
\text { Peak Pressure }\left({ }^{\circ} \mathrm{CA}\right)\end{array}$} \\
\hline & HF & RG & HF & RG & HF & RG & HF & RG \\
\hline $6500 \mathrm{r} / \mathrm{min}$ & 387.27 & 350.84 & 448.51 & 379.25 & 28.65 & 28.85 & 14.84 & 14.78 \\
\hline $6000 \mathrm{r} / \mathrm{min}$ & 383.86 & 349.84 & 443.23 & 378.31 & 29.54 & 29.97 & 14.91 & 14.93 \\
\hline $5500 \mathrm{r} / \mathrm{min}$ & 391.15 & 358.02 & 447.71 & 385.66 & 30.24 & 30.68 & 14.99 & 15.05 \\
\hline $5000 \mathrm{r} / \mathrm{min}$ & 393.21 & 359.19 & 449.38 & 389.52 & 30.45 & 30.90 & 14.93 & 14.96 \\
\hline $4500 \mathrm{r} / \mathrm{min}$ & 388.63 & 357.46 & 445.63 & 388.63 & 31.27 & 31.70 & 15.04 & 15.08 \\
\hline $4000 \mathrm{r} / \mathrm{min}$ & 392.01 & 365.63 & 450.72 & 394.38 & 31.59 & 32.08 & 14.92 & 14.99 \\
\hline $3500 \mathrm{r} / \mathrm{min}$ & 406.22 & 370.93 & 453.17 & 396.99 & 30.53 & 30.97 & 14.91 & 14.89 \\
\hline $3000 \mathrm{r} / \mathrm{min}$ & 409.86 & 382.37 & 459.97 & 406.32 & 32.58 & 33.05 & 15.12 & 15.10 \\
\hline
\end{tabular}

Table 19. Simulation results of engine load characteristics (engine speed $=6500 \mathrm{r} / \mathrm{min}$ ).

\begin{tabular}{ccccccccc}
\hline \multirow{2}{*}{$\begin{array}{c}\text { Engine Load } \\
\text { (Throttle Valve Opening) }\end{array}$} & \multicolumn{2}{c}{$\begin{array}{c}\text { Brake Power } \\
(\mathbf{k W})\end{array}$} & \multicolumn{2}{c}{$\begin{array}{c}\text { Brake Torque } \\
\mathbf{( N \cdot m )}\end{array}$} & \multicolumn{2}{c}{$\begin{array}{c}\text { IMEP } \\
\text { (bar) }\end{array}$} & \multicolumn{2}{c}{$\begin{array}{c}\text { BMEP } \\
\text { (bar) }\end{array}$} \\
\cline { 2 - 10 } & HF & RG & HF & RG & HF & RG & HF & RG \\
\hline $100 \%$ & 24.10 & 28.48 & 35.41 & 41.84 & 5.20 & 5.74 & 4.49 & 5.31 \\
$60 \%$ & 22.59 & 26.86 & 33.19 & 39.46 & 4.95 & 5.45 & 4.34 & 5.01 \\
$45 \%$ & 20.57 & 24.71 & 30.22 & 36.31 & 4.55 & 5.12 & 3.95 & 4.61 \\
$35 \%$ & 17.60 & 21.58 & 25.85 & 31.7 & 3.98 & 4.57 & 3.38 & 4.03 \\
$30 \%$ & 15.68 & 19.55 & 23.04 & 28.72 & 3.60 & 4.11 & 3.01 & 3.65 \\
$25 \%$ & 13.09 & 16.81 & 19.23 & 24.69 & 3.10 & 3.58 & 2.51 & 3.14 \\
$20 \%$ & 10.73 & 14.32 & 15.77 & 21.04 & 2.64 & 3.09 & 2.06 & 2.67 \\
$10 \%$ & 6.35 & 9.70 & 9.33 & 14.25 & 1.79 & 2.20 & 1.22 & 1.81 \\
\hline
\end{tabular}

Table 20. Simulation results of engine load characteristics (engine speed $=6500 \mathrm{r} / \mathrm{min}$ ).

\begin{tabular}{ccccccccc}
\hline $\begin{array}{c}\text { Engine Load } \\
\text { (Throttle Valve Opening) }\end{array}$ & \multicolumn{2}{c}{$\begin{array}{c}\text { ISFC } \\
\mathbf{g} / \mathbf{k W} \cdot \mathbf{h})\end{array}$} & \multicolumn{2}{c}{$\begin{array}{c}\text { BSFC } \\
\mathbf{g} / \mathbf{k W} \cdot \mathbf{h})\end{array}$} & \multicolumn{2}{c}{$\begin{array}{c}\text { Peak Pressure } \\
\mathbf{( b a r )}\end{array}$} & \multicolumn{2}{c}{$\begin{array}{c}\text { Crank Angle at the Peak } \\
\text { Pressure }\left({ }^{\circ} \mathbf{C A}\right)\end{array}$} \\
\cline { 2 - 9 } & HF & RG & HF & RG & HF & RG & HF & RG \\
\hline $100 \%$ & 387.27 & 350.84 & 448.51 & 379.25 & 28.65 & 28.85 & 14.84 & 14.78 \\
$60 \%$ & 376.37 & 341.84 & 429.27 & 371.86 & 27.52 & 27.72 & 14.72 & 14.69 \\
$45 \%$ & 383.45 & 340.76 & 441.69 & 378.46 & 26.12 & 26.29 & 14.45 & 14.46 \\
$35 \%$ & 386.19 & 336.34 & 454.75 & 381.40 & 24.17 & 24.30 & 14.01 & 14.13 \\
$30 \%$ & 393.75 & 344.89 & 470.93 & 388.36 & 22.93 & 23.05 & 13.70 & 13.79 \\
$25 \%$ & 397.75 & 344.42 & 491.25 & 392.69 & 21.24 & 21.33 & 13.22 & 13.28 \\
$20 \%$ & 405.27 & 346.25 & 519.38 & 400.72 & 19.65 & 19.72 & 12.57 & 12.63 \\
$10 \%$ & 379.23 & 308.55 & 556.41 & 375.04 & 16.78 & 16.82 & 10.87 & 11.03 \\
\hline
\end{tabular}


As displayed in Tables 17 and 18, all of these evaluation indicators of power performance (i.e., brake power, brake torque, IMEP, BMEP) drop remarkably when the initial prototype engine is fueled with heavy fuel instead of regular gasoline, which further corroborates the so-called "power attenuation" problem. This result slightly differs from the conclusion drawn by Hooper et al. [44]. With respect to the evaluation indicators of fuel economy performance, it can be observed that both ISFC and BSFC are significantly higher when the initial prototype engine is supplied with heavy fuel. As described above, low volatility and high viscosity make the injected heavy fuel more difficult to form the homogeneous air-fuel mixture. With less fresh charge of air-fuel mixture sent into the cylinder, the engine consequently outputs lower power and torque. As for the combustion characteristics, only subtle differences in the simulation results can be noticed when the supplied fuel is switched from regular gasoline to heavy fuel.

Similar conclusions can be drawn based on the comparative simulation results shown in Tables 19 and 20. Under different operation conditions of engine load, each simulation case proves that the usage of heavy fuel results in the power decrease and fuel consumption increase simultaneously. In general, the deterioration of performance characteristics of the initial prototype engine is virtually unavoidable without corresponding retrofit about the fuel injection system, which also validates the necessity for converting conventional PFI system into novel fuel injection system such as CWI system and SPSDI system when the engine is supplied with heavy fuel.

\subsection{Performance Comparison of the Three Types of Engines}

Based on the pre-established simulation platforms of all the three types of engine, comparative simulation is also performed. As mentioned in Section 3.2, the running status of engines on the small and medium-sized UAVs is generally characterized by three typical operation conditions including the minimum load, the medium load, and the full load. Therefore, simulation cases are configured and implemented under the three above-mentioned operation conditions. For the purpose of verifying the performance improvement of the retrofitted engine, the type of supplied fuel is set to heavy fuel in all simulation cases. Comparative simulation results are shown in Tables $21-23$ as follows:

Table 21. Comparative simulation results of the minimum load operation condition (heavy fuel).

\begin{tabular}{cccc}
\hline Performance Indicators & Initial Prototype Engine & Reference Engine & Retrofitted Engine \\
\hline Brake Power $(\mathrm{kW})$ & 7.85 & 8.02 & 8.11 \\
Brake Torque $(\mathrm{N} \cdot \mathrm{m})$ & 16.66 & 17.02 & 17.21 \\
IMEP/BMEP $(\mathrm{bar})$ & $2.59 / 2.11$ & $2.62 / 2.16$ & $2.63 / 2.18$ \\
ISFC/BSFC $(\mathrm{g} / \mathrm{kW} \cdot \mathrm{h})$ & $425.59 / 522.38$ & $413.82 / 501.95$ & $410.03 / 494.67$ \\
Peak Pressure $(\mathrm{bar})$ & 18.59 & 20.37 & 20.61 \\
Crank Angle at the Peak & 14.64 & 10.55 & 10.08 \\
Pressure $\left({ }^{\circ} \mathrm{CA}\right)$ & & & \\
\hline
\end{tabular}

Table 22. Comparative simulation results of the medium load operation condition (heavy fuel).

\begin{tabular}{cccc}
\hline Performance Indicators & Initial Prototype Engine & Reference Engine & Retrofitted Engine \\
\hline Brake Power $(\mathrm{kW})$ & 20.88 & 21.25 & 21.33 \\
Brake Torque $(\mathrm{N} \cdot \mathrm{m})$ & 34.38 & 34.99 & 35.12 \\
IMEP/BMEP $(\mathrm{bar})$ & $4.89 / 4.36$ & $4.95 / 4.44$ & $4.97 / 4.46$ \\
ISFC/BSFC $(\mathrm{g} / \mathrm{kW} \cdot \mathrm{h})$ & $372.11 / 417.34$ & $367.42 / 409.62$ & $359.64 / 400.77$ \\
Peak Pressure $(\mathrm{bar})$ & 26.41 & 29.29 & 29.56 \\
Crank Angle at the Peak & 14.94 & 10.98 & 10.27 \\
Pressure $\left({ }^{\circ} \mathrm{CA}\right)$ & & & \\
\hline
\end{tabular}


Table 23. Comparative simulation results of the full load operation condition (heavy fuel).

\begin{tabular}{cccc}
\hline Performance Indicators & Initial Prototype Engine & Reference Engine & Retrofitted Engine \\
\hline Brake Power $(\mathrm{kW})$ & 24.10 & 24.42 & 24.66 \\
Brake Torque $(\mathrm{N} \cdot \mathrm{m})$ & 35.41 & 35.88 & 36.23 \\
IMEP/BMEP $(\mathrm{bar})$ & $5.20 / 4.49$ & $5.24 / 4.55$ & $5.27 / 4.60$ \\
ISFC/BSFC $(\mathrm{g} / \mathrm{kW} \cdot \mathrm{h})$ & $387.27 / 448.51$ & $381.19 / 439.13$ & $373.33 / 428.35$ \\
Peak Pressure $(\mathrm{bar})$ & 28.65 & 32.06 & 32.75 \\
Crank Angle at the Peak & 14.84 & 10.96 & 10.21 \\
Pressure $\left({ }^{\circ} \mathrm{CA}\right)$ & & & \\
\hline
\end{tabular}

Taking the minimum load operation condition as an example, an obvious conclusion that can be drawn is that both the reference engine and the retrofitted engine show better performance over the initial prototype engine. With respect to these two, the performance improvement of the retrofitted engine is slightly greater than that of the reference engine. In terms of power performance, the brake power/brake torque/BMEP of the retrofitted engine are 3.3\% higher than that of the initial prototype engine, while the reference engine offers an improvement of $2.1 \%$. As for fuel economy performance, the BSFC of the retrofitted engine is 5.3\% lower than that of the initial prototype engine, while the reference engine offers a reduction of $3.9 \%$.

What calls for special attention is that the peak pressure of the retrofitted engine is significantly higher than that of the initial prototype engine. Additionally, the crank angle at the peak pressure of the retrofitted engine is closer to TDC $\left(0^{\circ} \mathrm{CA}\right)$ than that of the initial prototype engine. With both factors taken into consideration, it can be concluded that the fuel burning process is promoted more sufficiently, and the heat release process is more concentrated around TDC in the retrofitted engine compared with the initial prototype engine, which consequently leads to the improvement of the retrofitted engine in the aspect of above-mentioned performance characteristics.

Similar conclusions can be drawn with respect to the other two operation conditions, thus there is no more detailed description. Combined with the results of preliminary analysis shown in Table 4, comparative simulation results demonstrate that the retrofitted engine shows an improvement over the initial prototype engine in terms of important evaluation indicators of performance characteristics, and its improvement effect is more significant than that of the reference engine.

For the reference engine, fuel is injected into the long and thin CWI tube, which provides strong potential for the injected fuel to pool. However, fuel is directly injected into the cylinder and then vaporized in the retrofitted engine, so that the fuel pooling problem is eliminated. Considering that the application of the reference engine is heavily restricted by high mechanical processing difficulty and fuel pooling problem while the retrofitted engine gets rid of these deficiencies structurally, the unique advantages of the retrofitted engine in power performance, fuel economy performance, practicality, and manufacturability are confirmed by comprehensive analysis.

\section{Discussion}

Rapid advances in modern technologies have significantly promoted the development of small and medium-sized UAVs. Because of its significant advantages on power density and the safety of supplied fuel, 2SHFLA has simultaneously drawn extensive attention from both aviation industry and academia in the wake of research focus on the power systems for small and medium-sized UAVs. To address the "power attenuation" problem, necessary modifications about the fuel injection system need to be made, and 2SHFLA with different fuel injection systems is consequently selected as the study object in this paper.

With respect to the specialized fuel injection system for 2SHFLA, previous studies have failed to provide satisfactory design scheme. The AADI system developed by Orbital Engine Company must be used with compressed air source and pressure regulator as auxiliary devices, which sets barriers to lightweight design of 2SHFLA. In addition, the reliability of these sophisticated devices is relatively 
low when UAVs driven by 2SHFLA with an AADI system are flying at high altitude. The CWI system proposed by William of the Design \& Manufacturing Solutions Inc. addresses some deficiencies of AADI system, but it also brings about new problems such as fuel pooling and the high processing difficulty of the connecting orifice.

Based on the designing concept of the CWI system, a novel engine scheme of 2SHFLA with the SPSDI system (i.e., the retrofitted engine) is proposed, and its working principle is elucidated in detail. By dividing the initial prototype engine into several subsystems, the integrated simulation platform is gradually constructed in GT-ISE with its modeling process adequately described. The experiment platform of the initial prototype engine is also constructed and relevant tests under typical operation conditions are carried out to lay a solid foundation for accurate calibration. With these experimental data points as the criteria, these important model parameters are fine-tuned within the appropriate range, and thus the simulation precision can be guaranteed.

By making modifications about fuel injection system, the simulation platforms of the other two types of engine are also established. Subsequently, corresponding simulation is conducted, and the simulation results of all the three types of engine are compared.

\section{Conclusions}

It should be noticed that the established simulation platforms suffer from limitations because of some simplifications made in the modeling process. Despite that inadequacy, the present research findings are able to provide both practicable technical solution and requisite simulation data necessary for the performance improvement of 2SHFLA. Hopefully, the proposed designing scheme of the retrofitted engine with the SPSDI system can be employed as the reference idea for developing advanced power system of small and medium UAVs. Comprehensive analysis leads to the following conclusions:

(1) A preliminary comparison of the characteristics of all the three engines shows that the retrofitted engine has distinct advantages in many aspects such as the resonant intake system which offers considerable potential for increasing inlet flux, relatively high atomization quality of heavy fuel, and neither high mechanical processing difficulty nor a fuel pooling problem.

(2) The modular modeling method provided by GT-SUITE can be adopted to establish the integrated simulation platforms of all the three engines. By dividing the engine into different subsystems, each part can be easily modeled using the dedicated simulation module. The graphical modeling simulation environment GT-ISE can not only shorten the modeling time, promote the modeling efficiency, but also improve system reliability, flexibility, transferability, and expandability.

(3) To guarantee the accuracy of the established simulation platforms, the crucial parameter settings of corresponding models are calibrated against the obtained experimental data points, which also set the stage for subsequent comparative simulation.

(4) Simulation results show that significant performance degradation (i.e., lower power, more fuel consumption) can be observed when the initial prototype engine is fueled with heavy fuel instead of regular gasoline. In addition, the retrofitted engine with the SPSDI system shows its superiority in performance evaluation indicators of both power and fuel economy over the other two types of engines (In terms of power performance, the brake power/brake torque/BMEP of the retrofitted engine are $3.3 \%$ higher than that of the initial prototype engine, while the reference engine offers an improvement of $2.1 \%$. As for fuel economy performance, the BSFC of the retrofitted engine is $5.3 \%$ lower than that of the initial prototype engine, while the reference engine offers a reduction of $3.9 \%$ ), which consequently verifies the feasibility of this proposed engine designing scheme.

Author Contributions: Conceptualization, K.H. and Y.Q.; Methodology, Y.Q. and L.L.; Software, Y.Q.; Formal Analysis, Y.Q. and L.L.; Data Curation, Y.Q. and W.Z.; Writing-Original Draft Preparation, Y.Q.; Writing-Review and Editing, K.H. and Y.Q. All authors have read and agreed to the published version of the manuscript.

Funding: This research was funded by National Key Research and Development Program Grant No. 2016 YFB0101402. 
Acknowledgments: The authors gratefully acknowledge the technical support from Alternative Powertrain Solutions (Tianjin) Co., Ltd.

Conflicts of Interest: The authors declare no conflict of interest.

\section{Abbreviation}

$\begin{array}{ll}\text { UAVs } & \text { unmanned aerial vehicles } \\ \text { 2SHFLA } & \text { 2-stroke heavy fuel light aeroengine } \\ \text { AADI } & \text { air assisted direct injection } \\ \text { MPI } & \text { multi-point port injection } \\ \text { TDC } & \text { top dead center } \\ \text { ISFC } & \text { indicated specific fuel consumption } \\ \text { BSFC } & \text { brake specific fuel consumption } \\ \text { 0-D } & \text { zero-dimensional } \\ \text { 3-D } & \text { three-dimensional } \\ \text { CFD } & \text { computational fluid dynamics } \\ \text { SMD } & \text { Sauter mean diameter } \\ \text { PFI } & \text { port fuel injection } \\ \text { CWI } & \text { compression wave injection } \\ \text { SPSDI } & \text { scavenging port semi-direct injection } \\ \text { GDI } & \text { gasoline direct injection } \\ \text { OEM } & \text { original equipment manufacturer } \\ \text { FMEP } & \text { friction mean effective pressure } \\ \text { IMEP } & \text { indicated mean effective pressure } \\ \text { BMEP } & \text { brake mean effective pressure } \\ \text { ECU } & \text { electronic control unit } \\ \text { FSR } & \text { full scale range } \\ \text { SV } & \text { simulation value } \\ \text { EV } & \text { experiment value } \\ \text { RG } & \text { regular gasoline } \\ \text { HF } & \text { heavy fuel } \\ & \end{array}$

\section{Notation}

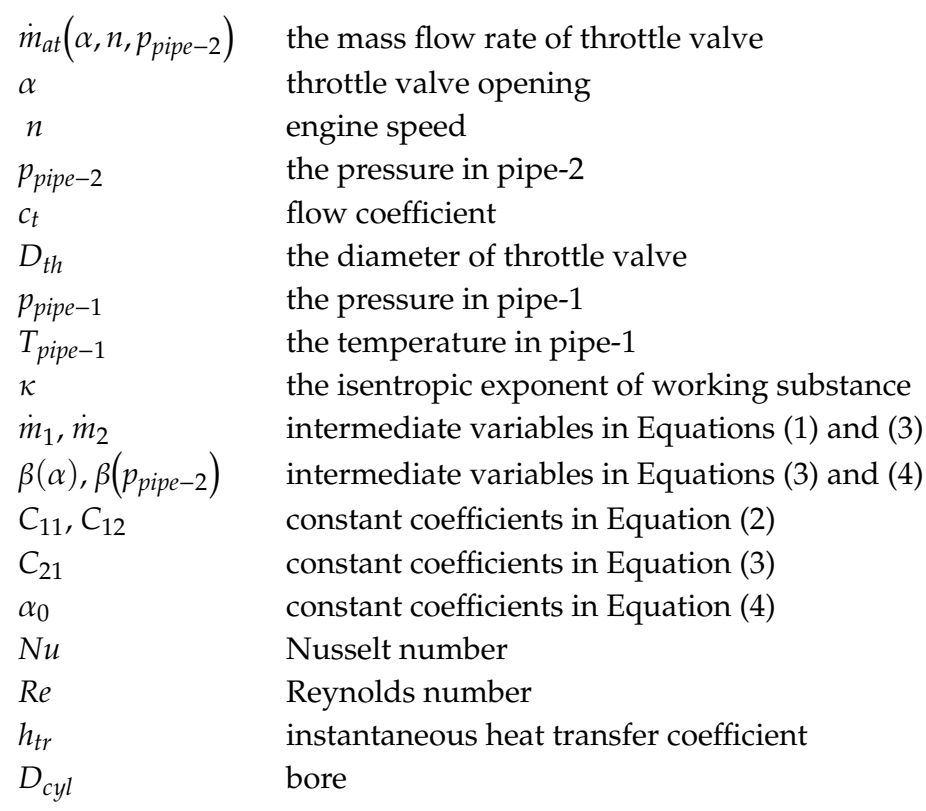




\begin{tabular}{|c|c|}
\hline$v_{p m}$ & mean piston speed \\
\hline$p_{c y l}$ & cylinder pressure \\
\hline$T_{c y l}$ & cylinder temperature \\
\hline$C_{f v}$ & flow velocity coefficient \\
\hline$C_{c c s}$ & the coefficient of combustion chamber shape \\
\hline$T_{\mathcal{C S}}$ & the temperature at the start of compression stroke \\
\hline$p_{c S}$ & the cylinder pressure at the start of compression stroke \\
\hline$V_{c S}$ & the cylinder volume at the start of compression stroke \\
\hline$V_{S}$ & the displacement of a single cylinder \\
\hline$p_{m o}$ & the cylinder pressure under the motored condition \\
\hline FMEP $_{\text {const }}$ & the constant pressure term of FMEP in Equation (8) \\
\hline$A_{F}$ & peak cylinder pressure factor \\
\hline$B_{F}$ & mean piston speed factor \\
\hline$C_{F}$ & mean piston speed squared factor \\
\hline$p_{c y l, \max }$ & maximum cylinder pressure \\
\hline$S$ & stroke \\
\hline$A A$ & anchor angle \\
\hline$C D$ & combustion duration \\
\hline$W E$ & Wiebe exponent \\
\hline$B S$ & the burned fuel percentage at duration start \\
\hline$B M$ & the burned fuel percentage at anchor angle \\
\hline$B E$ & the burned fuel percentage at duration end \\
\hline$B S C$ & burned start constant \\
\hline$B M C$ & burned midpoint constant \\
\hline$B E C$ & burned end constant \\
\hline$W C$ & Wiebe constant \\
\hline SOC & the start of combustion \\
\hline Combustion $(\varphi)$ & cumulative burn rate \\
\hline$\varphi$ & instantaneous crank angle \\
\hline$F F B$ & the fraction of fuel burned \\
\hline$\varepsilon_{g e o}$ & geometric compression ratio \\
\hline$V_{c c}$ & the volume of combustion chamber on the cylinder head \\
\hline$l_{s c}$ & the length of scavenging ports \\
\hline$h_{s c}$ & the distance from TDC surface to scavenging ports \\
\hline$l_{e x}$ & the length of exhaust port \\
\hline$h_{e x}$ & the distance from TDC surface to exhaust port \\
\hline EOC & the end of combustion \\
\hline$Q_{\text {all }}$ & the total heat release from $S O C$ to $E O C$ \\
\hline$d Q_{b}$ & heat release rate \\
\hline$\alpha_{c c}$ & the heat transfer coefficient of combustion chamber wall \\
\hline$F_{c c}$ & the heat transfer area of combustion chamber wall \\
\hline$T_{c c}$ & the temperature of combustion chamber wall \\
\hline$\eta_{V}$ & volumetric efficiency \\
\hline$\rho_{\text {ref }}$ & the reference density needed for calculating $\eta_{V}$ \\
\hline$A / F$ & the imposed air-fuel ratio \\
\hline$\dot{m}_{i n j}$ & the delivery rate of injector \\
\hline
\end{tabular}


Appendix A

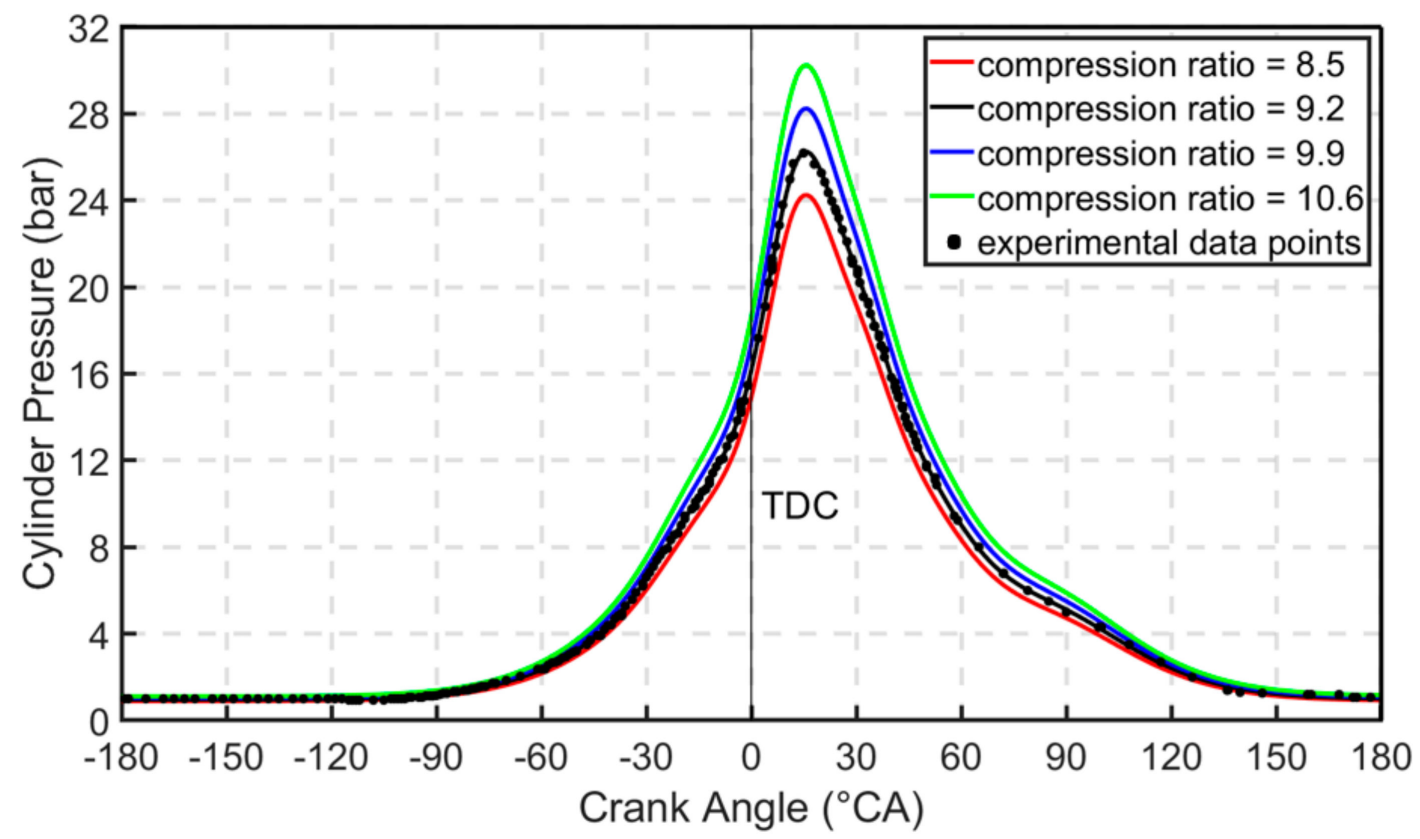

Figure A1. Comparison between cylinder curves with different compression ratios (medium load).

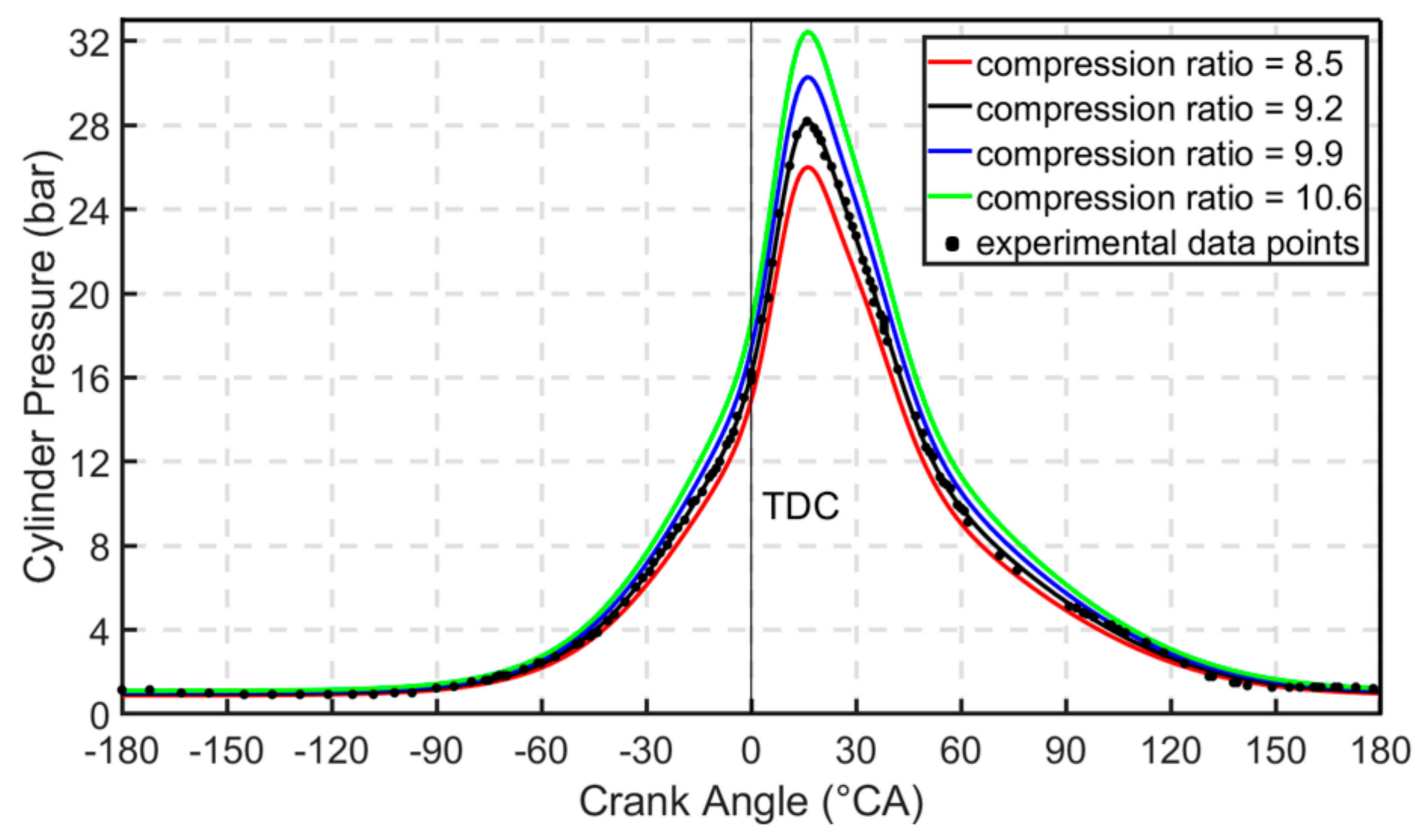

Figure A2. Comparison between cylinder curves with different compression ratios (full load). 


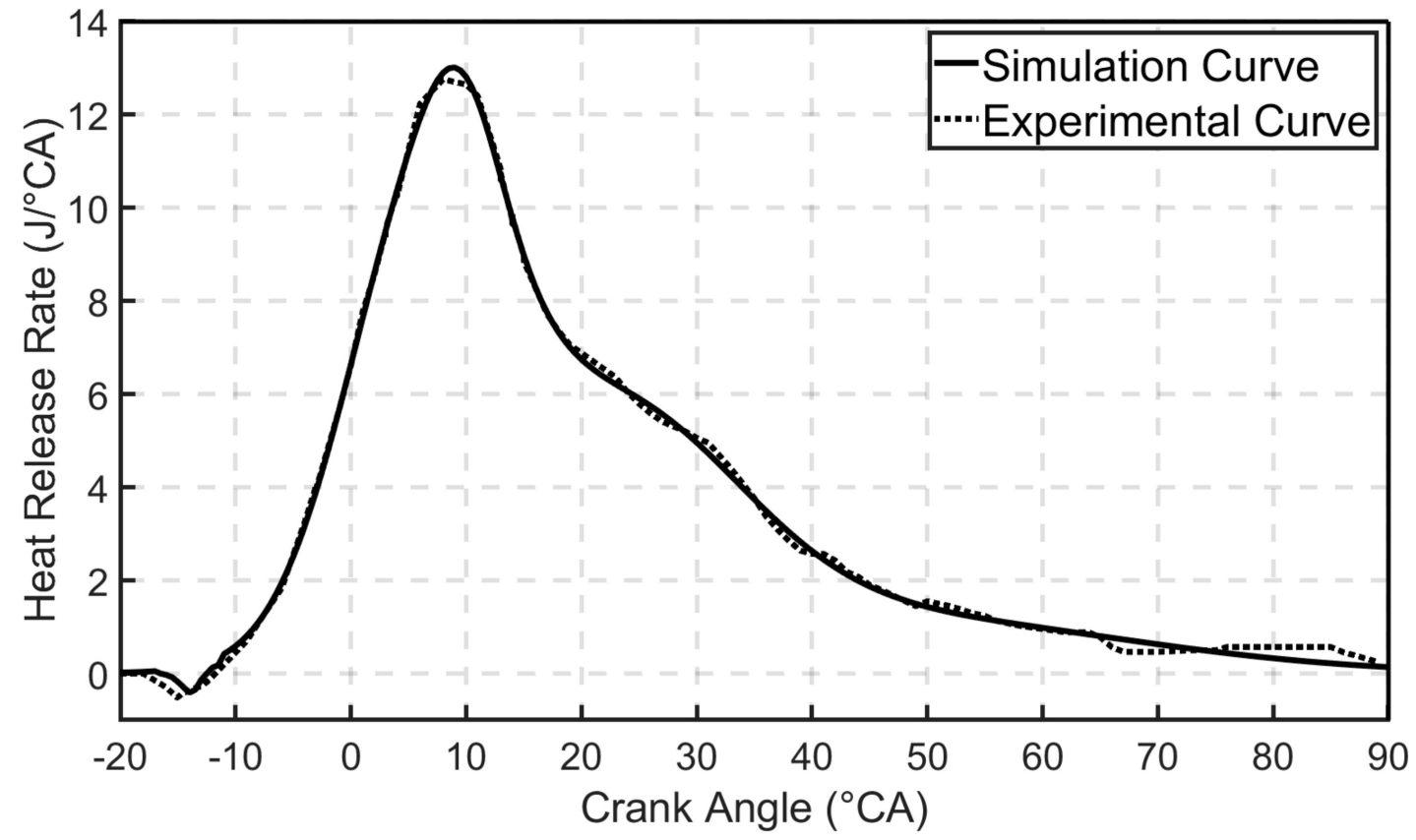

Figure A3. Comparison between heat release curves (medium load).

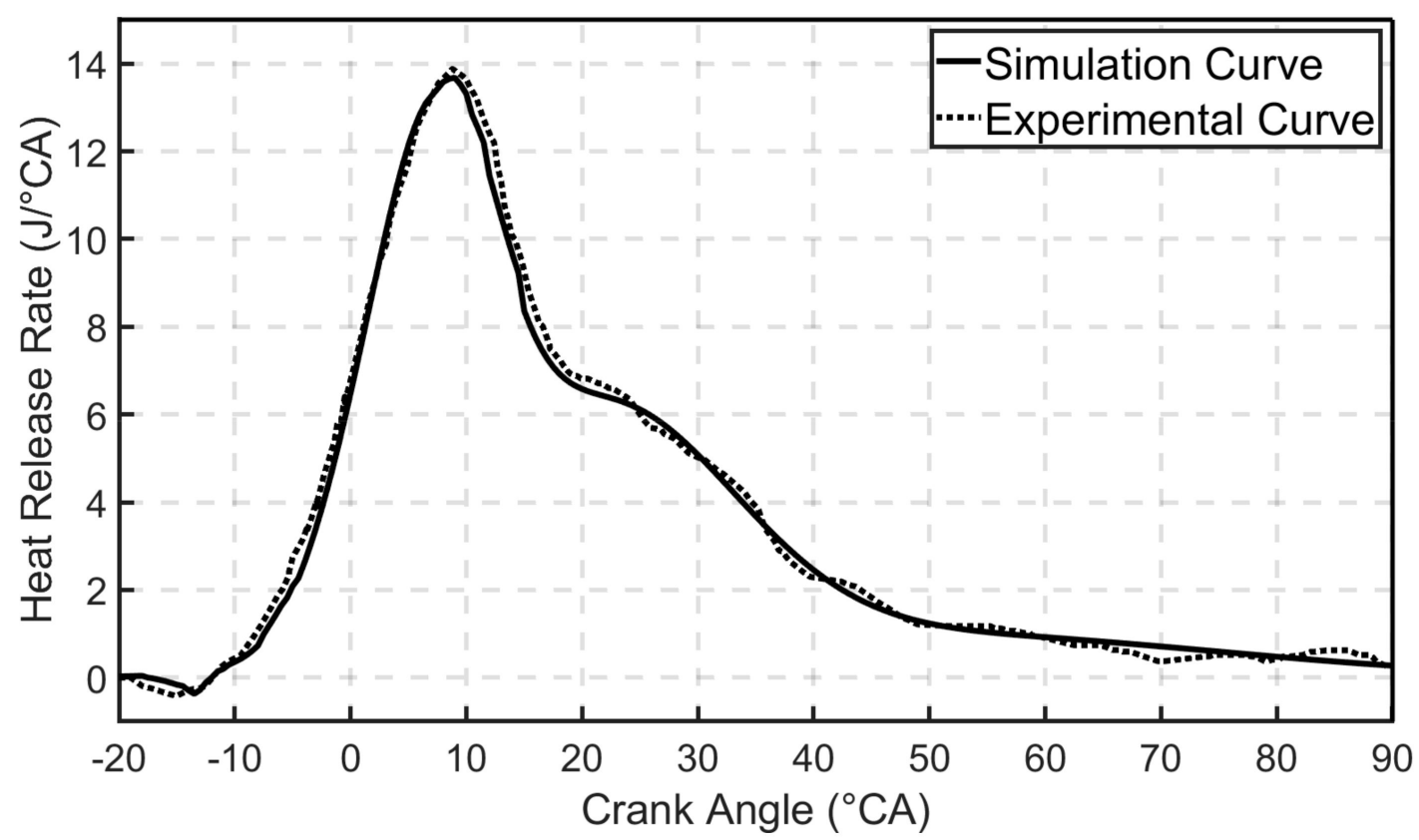

Figure A4. Comparison between heat release curves (full load).

\section{References}

1. Finger, D.F.; Braurr, C.; Bil, C. Case studies in initial sizing for hybrid-electric general aviation aircraft. In Proceedings of the 2018 AIAA/IEEE Electric Aircraft Technologies Symposium (EATS), Cincinnati, OH, USA, 9-11 July 2018; pp. 1-22.

2. Aldridge, E.C.; Stenbit, J.P. Unmanned Aerial Vehicles Roadmap 2002-2027; Department of Defense USA: Washington, DC, USA, 2002.

3. Thiels, C.A.; Aho, J.M.; Zietlow, S.P.; Jenkins, D.H. Use of unmanned aerial vehicles for medical product transport. Air Med. J. 2015, 34, 104-108. [CrossRef] [PubMed] 
4. Zarándy, Á.; Zsedrovits, T.; Nagy, Z.; Kiss, A.; Roska, T. Visual sense-and-avoid system for UAVs. In Proceedings of the 2012 13th International Workshop on Cellular Nanoscale Networks and their Applications (CNNA), Turin, Italy, 29-31 August 2012; pp. 1-5.

5. Cruzan, M.B.; Weinstein, B.G.; Grasty, M.R.; Kohrn, B.F.; Hendrickson, E.C.; Arredondo, T.M.; Thompson, P.G. Small unmanned aerial vehicles (micro-UAVs, drones) in plant ecology. Appl. Plant. Sci. 2016, 4, 1600041. [CrossRef] [PubMed]

6. Crosbie, S.; Polanka, M.; Litke, P.; Hoke, J. Increasing reliability of a small 2-stroke internal combustion engine for dynamically changing altitudes. In Proceedings of the 50th AIAA Aerospace Sciences Meeting Including the New Horizons Forum and Aerospace Exposition, Nashville, TN, USA, 9-12 January 2012; p. 950.

7. Sousa, J.; Paniagua, G.; Morata, E.C. Thermodynamic analysis of a gas turbine engine with a rotating detonation combustor. Appl. Energy 2017, 195, 247-256. [CrossRef]

8. Bhangu, B.S.; Rajashekara, K. Control strategy for electric starter generators embedded in gas turbine engine for aerospace applications. In Proceedings of the IEEE Energy Conversion Congress and Exposition (ECCE), Phoenix, AZ, USA, 17-22 September 2011; pp. 1461-1467.

9. Cirigliano, D. Engine-Type and Propulsion Configuration Selections for Long-Duration UAV Flights. Master's Thesis, University of California, Irvine, CA, USA, 20 March 2017.

10. Capata, R.; Marino, L.; Sciubba, E. A hybrid propulsion system for a high-endurance UAV: Configuration selection, aerodynamic study, and gas turbine bench tests. J. Unmanned Veh. Syst. 2014, 2, 16-35. [CrossRef]

11. Husaboe, T.D.; Polanka, M.D.; Rittenhouse, J.A.; Litke, P.J.; Hoke, J.L. Dependence of Small Internal Combustion Engine's Performance on Altitude. J. Propuls. Power 2014, 30, 1328-1333. [CrossRef]

12. Hooper, P. Low Volatility Fuel Cold Start Experience with a Stepped Piston UAV Engine to Address Single Fuel Objectives; Technical Paper; SAE International: Detroit, MI, USA, 2017.

13. Duddy, B.J.; Lee, J.; Walluk, M.; Hallbach, D. Conversion of a spark-ignited aircraft engine to JP-8 heavy fuel for use in unmanned aerial vehicles. SAE Int. J. Engines 2011, 4, 82-93. [CrossRef]

14. Liu, R.; Wei, M.; Wang, C.; Huang, T. Fuel Flow Control for Starting a Crankcase-Injected Two-Stroke Spark Ignition Engine Fueled with Kerosene (RP-3). J. Energy Eng. 2019, 145, 04019010. [CrossRef]

15. Feng, G.; Zhou, M. Assessment of heavy fuel aircraft piston engine types. J. Tsinghua Univ. 2016, 56, 1114-1121.

16. Hooper, P. Experimental experience of cold starting a spark ignition UAV engine using low volatility fuel. Aircr. Eng. Aerosp. Technol. 2017, 89, 106-111. [CrossRef]

17. Ma, S.; Li, N.; Hu, C. Present Status and Development Direction of Two Stroke Heavy Fuel Engine. Small Intern. Combust. Engine Veh. Tech. 2015, 44, 87-91.

18. Gao, L.; Wang, H.; He, K. Application of Aviation Heavy Oil in Aviation Piston Engine. Small Intern. Combust. Engine Veh. Tech. 2018, 47, 84-89.

19. Leighton, S.; Cebis, M.; Southern, M.; Ahern, S.; Horner, L. The OCP Small Engine Fuel Injection System for Future Two-Stroke Marine Engines; Technical Paper; SAE International: Detroit, MI, USA, 1994.

20. Worth, D.; Coplin, N.; McNiff, M.; Stannard, M. Design Considerations for the Application of Air Assisted Direct In-Cylinder Injection Systems; Technical Paper; SAE International: Detroit, MI, USA, 1997.

21. Houston, R.; Cathcart, G. Combustion and Emissions Characteristics of Orbital's Combustion Process Applied to Multi-Cylinder Automotive Direct Injected 4-Stroke Engines; Technical Paper; SAE International: Detroit, MI, USA, 1998.

22. Cathcart, G.; Zavier, C. Fundamental Characteristics of an Air-Assisted Direct Injection Combustion System as Applied to 4-Stroke Automotive Gasoline Engines; Technical Paper; SAE International: Detroit, MI, USA, 2000.

23. Cathcart, G.; Dickson, G.; Ahern, S. The Application of Air-Assist Direct Injection for Spark-Ignited Heavy Fuel 2-Stroke and 4-Stroke Engines; Technical Paper; SAE International: Detroit, MI, USA, 2005.

24. Groenewegen, J.R.; Sidhu, S.; Hoke, J.; Wilson, C.; Litke, P. The performance and emissions effects of utilizing heavy fuels and algae based biodiesel in a port-fuel-injected small spark ignition internal combustion engine. In Proceedings of the 47th AIAA/ASME/SAE/ASEE Joint Propulsion Conference \& Exhibit, San Diego, CA, USA, 31 July-3 August 2012; p. 5807.

25. Koci, C.; Florea, R.; Das, S.; Walls, M.; Simescu, S.; Roberts, C. Air-Assisted Direct Injection Diesel Investigations; Technical Paper; SAE International: Detroit, MI, USA, 2013.

26. La, C.; Murphy, P.; Cakebread, S. Benchmarking a 2-Stroke Spark Ignition Heavy Fuel Engine; Technical Paper; SAE International: Detroit, MI, USA, 2012. 
27. Gao, H.; Zhang, F.; Wang, S. Experimental Study of Air-assisted Spray Characteristics of Aviation Kerosene Piston Engine. Acta Armamentarii 2019, 40, 927-937.

28. Yang, H.; Chen, M.; Huang, L.; Hu, C. CFD simulation and experiment of transient spray for an air-assisted injector. J. Aerosp. Power 2015, 30, 2897-2903.

29. Wang, S.; Yang, H. Simulation on Atomization Mechanism of Air-Assisted Injector for Aircraft Heavy Fuel Engine. Trans. CSICE 2018, 36, 127-135.

30. Hu, C.; Wang, S.; Bi, Y.; Zhong, W. Combustion characteristics of direct injection piston aviation kerosene engine. J. Aerosp. Power 2017, 32, 1035-1042.

31. Qiao, Y.; Duan, X.; Huang, K.; Song, Y.; Qian, J. Scavenging Ports' Optimal Design of a Two-Stroke Small Aeroengine Based on the Benson/Bradham Model. Energies 2018, 11, 2739. [CrossRef]

32. Ma, F.K.; Wang, J.; Feng, Y.N.; Zhang, Y.G.; Su, T.X.; Zhang, Y.; Liu, Y.H. Parameter Optimization on the Uniflow Scavenging System of an OP2S-GDI Engine Based on Indicated Mean Effective Pressure (IMEP). Energies 2017, 10, 368. [CrossRef]

33. William, T.; Cobb, J. Compression Wave Injection: A Mixture Injection Method for Two-Stroke Engines Based on Unsteady Gas Dynamics; Technical Paper; SAE International: Detroit, MI, USA, 2001.

34. Hoffmann, G.; Befrui, B.; Berndorfer, A.; Piock, W.F.; Varble, D.L. Fuel system pressure increase for enhanced performance of GDI multi-hole injection systems. SAE Int. J. Engines 2014, 7, 519-527. [CrossRef]

35. Morel, T. Engine Performance Application Manual, GT-SUITE Version 2016; Gamma Technologies: Westmont, IL, USA, 2016.

36. GT-SUITE-A Revolutionary MBSE Tool. Available online: http://www.gtisoft.com/ (accessed on 19 July 2020).

37. Ouyang, M.; Li, J.; Yang, F.; Lu, L. Automotive New Powertrain: Systems, Models and Controls; Tsinghua University Press: Beijing, China, 2011.

38. Hooper, P.R.; Al-Shemmeri, T. Improved efficiency of an unmanned air vehicle IC engine using computational modelling and experimental verification. Aircr. Eng. Aerosp. Technol. 2017, 89, 184-192. [CrossRef]

39. Morel, T. Mechanics Theory Manual, GT-SUITE Version 2016; Gamma Technologies: Westmont, IL, USA, 2016.

40. Shuai, S.; Wang, J. Automotive Engine Fundamentals; Tsinghua University Press: Beijing, China, 2014.

41. Woschni, G. A Universally Applicable Equation for the Instantaneous Heat Transfer Coefficient in the Internal Combustion Engine; Technical Paper; SAE International: Detroit, MI, USA, 1967.

42. Wiebe, I. Semi-Empirical Formula for the Combustion Rate in Fuel Processing and Combustion in Diesel Engines; Springer: Berlin, Germany, 1964.

43. Chen, S.K.; Flynn, P.F. Development of a Single Cylinder Compression Ignition Research Engine; Technical Paper; SAE International: Detroit, MI, USA, 1965.

44. Hooper, P.R.; Al-Shemmeri, T.; Goodwin, M.J. An experimental and analytical investigation of a multi-fuel stepped piston engine. J. Appl. Therm. Eng. 2012, 48, 32-40. [CrossRef] 